\title{
Simplified neutrosophic sets and their applications in multi-criteria group decision-making problems
}

\author{
Juan-juan Peng ${ }^{\mathrm{a}, \mathrm{b}}$, Jian-qiang Wang $\odot^{\mathrm{a}, *}$, Jing Wang $\odot^{\mathrm{a}}$, Hong-yu Zhang $\odot^{\mathrm{a}}$ and Xiao-hong Chen $\odot^{\mathrm{a}}$ \\ ${ }^{a}$ School of Business, Central South University, Changsha, China; ${ }^{b}$ School of Economics and Management, Hubei University \\ of Automotive Technology, Shiyan, China
}

(Received 2 October 2013; accepted 12 February 2014)

\begin{abstract}
As a variation of fuzzy sets and intuitionistic fuzzy sets, neutrosophic sets have been developed to represent uncertain, imprecise, incomplete and inconsistent information that exists in the real world. Simplified neutrosophic sets (SNSs) have been proposed for the main purpose of addressing issues with a set of specific numbers. However, there are certain problems regarding the existing operations of SNSs, as well as their aggregation operators and the comparison methods. Therefore, this paper defines the novel operations of simplified neutrosophic numbers (SNNs) and develops a comparison method based on the related research of intuitionistic fuzzy numbers. On the basis of these operations and the comparison method, some SNN aggregation operators are proposed. Additionally, an approach for multi-criteria group decision-making (MCGDM) problems is explored by applying these aggregation operators. Finally, an example to illustrate the applicability of the proposed method is provided and a comparison with some other methods is made.
\end{abstract}

Keywords: simplified neutrosophic sets (SNSs); multi-criteria group decision-making (MCGDM); aggregation operators

\section{Introduction}

Zadeh (1965) proposed the theory of fuzzy sets (FSs) to solve different types of uncertainties. Since then, this theory has been applied successfully in various fields (Bellman \& Zadeh, 1970; Pedrycz, 1990; Yager, 1977; Zadeh, 1968, 1975). As the traditional FSs use one single value $\mu_{A}(x) \in[0,1]$ to represent the degree of membership of the fuzzy set $A$, which is defined on a universal scale, they cannot handle certain cases where it is hard to define $\mu_{A}$ by one specific value. In order to overcome the lack of knowledge of non-membership degrees, Atanassov introduced intuitionistic fuzzy sets (IFSs) (Atanassov, 1986, 1994, 1999, 2000), which are an extension of Zadeh's FSs. Moreover, Gau and Buehrer (1993) defined vague sets. Bustince and Burillo (1996) subsequently pointed out that these vague sets and Atanassov's IFSs are mathematically equivalent objects. To date, IFSs have been widely applied in solving multi-criteria decision-making (MCDM) problems (Chen, 2010; Liu \& Wang, 2007; Xu, 2012; Zeng \& Su, 2011; Zhi \& Li, 2012), neural networks (Sotirov, Sotirova, \& Orozova, 2009), medical diagnosis (Shinoj \& Sunil, 2012), colour region extraction (Chaira, 2010, 2011) and market prediction (Joshi \& Kumar, 2012).

IFSs take into account the membership degree, nonmembership degree and degree of hesitation simultaneously. Therefore, they are more flexible and practical when addressing fuzziness and uncertainty than traditional FSs.
Moreover, in some actual cases, the membership degree, non-membership degree and hesitation degree of an element in IFSs may be not a specific number. Hence, they were extended to interval-valued intuitionistic fuzzy sets (Atanassov \& Gargov, 1989). Furthermore, in order to handle the situations where people are hesitant when expressing their preferences regarding objects in the decision-making process, hesitant fuzzy sets were introduced by Torra and Narukawa (2009) and Torra (2010).

Although the FSs theory has been developed and generalised, it cannot deal with all uncertainty in different reallife problems. For instance, certain types of uncertainty, such as indeterminate and inconsistent information, cannot be dealt with. For example, when an expert is asked for his or her opinion about a certain statement, he or she may say that the possibility that the statement is true is 0.5 , that it is false is 0.6 and the degree that he or she is not sure is 0.2 (Wang, Smarandache, Zhang, \& Sunderraman, 2010). This issue is beyond the scope of FSs and IFSs, therefore some new theories are required.

Smarandache $(1999,2003)$ proposed neutrosophic logic and neutrosophic sets (NSs). An NS is a set where each element of the universe has the degrees of truth, indeterminacy and falsity and it lies in $] 0^{-}, 1^{+}[$, the nonstandard unit interval (Rivieccio, 2008). Clearly, this is an extension of the standard interval $[0,1]$ of IFSs. Moreover, the uncertainty presented here, i.e., the indeterminacy

\footnotetext{
*Corresponding author. Email: jqwang@csu.edu.cn
} 
factor, is independent of truth and falsity values, while the incorporated uncertainty is dependent on the degree of belongingness and non-belongingness of IFSs (Majumdar \& Samant, 2014). Furthermore, the aforementioned example of NSs can be expressed as $x(0.5,0.2,0.6)$.

However, without a specific description, NSs are difficult to apply in real-life situations. Hence, single-valued neutrosophic sets (SVNSs) were proposed, which are a variation of NSs (Majumdar \& Samant, 2014). Furthermore, the information energy of SVNSs, their correlation and correlation coefficient and the decision-making method that used them were also proposed (Ye, 2013). Additionally, Ye (2014a) also introduced the concept of simplified neutrosophic sets (SNSs), which can be described by three real numbers in the real unit interval $[0,1]$, and proposed an MCDM method using the aggregation operators of SNSs. Moreover, Majumdar and Samant (2014) introduced a measure of entropy of an SVNS. Wang, Smarandache, Zhang, and Sunderraman (2005) and Lupiáñez (2009) proposed the concept of interval neutrosophic sets (INSs) and proposed the set-theoretic operators of INSs. Furthermore, Ye (2013b, 2014c) proposed the similarity measures between SVNSs and INSs, which were based on the relationship between similarity measures and distances.

However, in some cases, the SNSs' operations (Ye, 2014a) may be impractical. For instance, the sum of any element and the maximum value should be equal to the maximum value, but this does not occur when using the SNSs' operations (Ye, 2014a). Therefore, the operations and comparison approach between simplified neutrosophic numbers (SNNs) and the aggregation operators for SNNs are re-defined in this paper. Moreover, a multi-criteria group decision-making (MCGDM) method is subsequently established based on the proposed operators.

The rest of paper is organised as follows. In Section 2, the properties of the t-norm and t-conorm as well as the concepts and operations of NSs and SNSs are briefly introduced. In Section 3, the operations and comparison approach for SNNs are defined on the basis of IFSs. In Section 4 , the aggregation operators of SNNs are provided and a decision-making method making use of them is developed for SNSs. In Section 5, an illustrative example is presented to test the proposed method, and a sensitivity analysis and comparison analysis are also provided. Finally, in Section 6, the conclusions are drawn.

\section{Preliminaries}

In this section, some basic concepts and definitions related to NSs, including the t-norm and t-conorm, and the definitions and operations of NSs and SNSs are introduced. All of these will be utilised in this paper.

\subsection{The t-norm and t-conorm}

The t-norm and its dual, the t-conorm, play an important role in constructing the operations and average operators of NSs. Here, some related basic concepts are introduced.

Definition 1 (Klement \& Mesiar, 2005; Nguyen \& Walker, 1997): A function $T:[0,1] \times[0,1] \rightarrow[0,1]$ is called a t-norm if it satisfies the following conditions:

(1) $\forall x \in[0,1], T(1, x)=x$;

(2) $\forall x, y \in[0,1], T(x, y)=T(y, x)$;

(3) $\forall x, y, z \in[0,1], T(x, T(y, z))=T(T(x, y), z)$;

(4) If $x \leq x^{\prime}, y \leq y^{\prime}$, then $T(x, y) \leq T\left(x^{\prime}, y^{\prime}\right)$.

Definition 2 (Klement \& Mesiar, 2005; Nguyen \& Walker, 1997): A function $S:[0,1] \times[0,1] \rightarrow[0,1]$ is called a tconorm if it satisfies the following conditions:

(1) $\forall x \in[0,1], S(0, x)=x$;

(2) $\forall x, y \in[0,1], S(x, y)=S(y, x)$;

(3) $\forall x, y, z \in[0,1], S(x, S(y, z))=S(S(x, y), z)$;

(4) If $x \leq x^{\prime}, y \leq y^{\prime}$, then $S(x, y) \leq S\left(x^{\prime}, y^{\prime}\right)$.

Definition 3 (Klement \& Mesiar, 2005; Nguyen \& Walker, 1997): A t-norm function $T(x, y)$ is called an Archimedean t-norm if it is continuous and $T(x, x)<x$ for all $x \in(0,1)$. An Archimedean t-norm is called a strictly Archimedean t-norm if it is strictly increasing in each variable for $x, y \in(0,1)$. A t-conorm function $S(x, y)$ is called an Archimedean t-conorm if it is continuous and $S(x, x)>x$ for all $x \in(0,1)$. An Archimedean t-conorm is called a strictly Archimedean t-conorm if it is strictly increasing in each variable for $x, y \in(0,1)$.

It is well known (Klement \& Mesiar, 2005; Klir \& Yuan, $1995)$ that a strict Archimedean t-norm is expressed via its additive generator $k$ as $T(x, y)=k^{-1}(k(x)+k(y))$, and similarly, applied to its dual t-conorm $S(x, y)=l^{-1}(l(x)+$ $l(y))$ with $l(t)=k(1-t)$. It has been observed that an additive generator of a continuous Archimedean t-norm is a strictly decreasing function $k:[0,1] \rightarrow[0, \infty)$.

There are also some Archimedean t-conorms and t-norms (Beliakov, Pradera, \& Calvo, 2007).

(1) Let $k(t)=-\log t, l(t)=-\log (1-t), k^{-1}(t)=$ $e^{-t}$ and $l^{-1}(t)=1-e^{-t}$. Then an algebraic tconorm and t-norm are obtained: $S(x, y)=1-$ $(1-x)(1-y)$ and $T(x, y)=x y$.

(2) Let $k(t)=\log \left(\frac{2-t}{t}\right), l(t)=\log \left(\frac{2-(1-t)}{1-t}\right), k^{-1}(t)=$ $\frac{2}{e^{t}+1}$ and $l^{-1}(t)=1-\frac{2}{e^{t}+1}$. Then an Einstein tconorm and t-norm are obtained: $S(x, y)=\frac{x+y}{1+x y}$ and $T(x, y)=\frac{x y}{1+(1-x)(1-y)}$. 


\subsection{NSs and SNSs}

In this section, the definitions and operations of NSs and SNSs are introduced.

Definition 4 (Smarandache, 1999): Let $X$ be a space of points (objects), with a generic element in $X$, denoted by $x$. An NS $A$ in $X$ is characterised by a truth-membership function $T_{A}(x)$, an indeterminacy-membership function $I_{A}(x)$ and a falsity-membership function $F_{A}(x) . T_{A}(x), I_{A}(x)$ and $F_{A}(x)$ are standard or non-standard subsets of $] 0^{-}, 1^{+}[$, that is, $\left.T_{A}(x): X \rightarrow\right] 0^{-}, 1^{+}\left[, I_{A}(x): X \rightarrow\right] 0^{-}, 1^{+}[$and $\left.F_{A}(x): X \rightarrow\right] 0^{-}, 1^{+}[$. There is no restriction on the sum of $T_{A}(x), I_{A}(x)$ and $F_{A}(x)$, therefore $0^{-} \leq \sup T_{A}(x)+$ $\sup I_{A}(x)+\sup F_{A}(x) \leq 3^{+}$.

Definition 5 (Smarandache, 1999): An NS $A$ is contained in another NS $B$, denoted by $A \subseteq B$, if and only if $\inf T_{A}(x) \leq \inf T_{B}(x), \sup T_{A}(x) \leq \sup T_{B}(x), \inf I_{A}(x) \geq$ $\inf I_{B}(x), \quad \sup I_{A}(x) \geq \sup I_{B}(x), \quad \inf F_{A}(x) \geq \inf F_{B}(x)$ and $\sup F_{A}(x) \geq \sup F_{B}(x)$ for $x \in X$.

Since it is difficult to apply NSs to practical problems, Ye (2014a) reduced NSs of non-standard intervals into SNSs of standard intervals that would preserve the operations of NSs.

Definition 6 (Ye, 2014a): Let $X$ be a space of points (objects), with a generic element in $X$, denoted by $x$. An NS $A$ in $X$ is characterised by $T_{A}(x), I_{A}(x)$ and $F_{A}(x)$, which are subintervals/subsets in the standard interval $[0,1]$, that is, $T_{A}(x): X \rightarrow[0,1], I_{A}(x): X \rightarrow[0,1]$ and $F_{A}(x):$ $X \rightarrow[0,1]$. Then, a simplification of $A$ is denoted by

$$
A=\left\{<x, T_{A}(x), I_{A}(x), F_{A}(x)>\mid x \in X\right\},
$$

which is called an SNS. In particular, if $\mathrm{X}$ has only one element, $A=<T_{A}(x), I_{A}(x), F_{A}(x)>$ is called an SNN. For convenience, an SNN is denoted by $A=<T_{A}, I_{A}, F_{A}>$. Clearly, SNSs are a subclass of NSs.

Definition 7 (Ye, 2014a): An SNS $A$ is contained in another SNS $B$, denoted by $A \subseteq B$, if and only if $T_{A}(x) \leq T_{B}(x)$, $I_{A}(x) \geq I_{B}(x)$ and $F_{A}(x) \geq F_{B}(x)$ for any $x \in X$.

The operations of SNSs are also defined in Ye (2014a).

Definition 8 (Ye, 2014a): Let $A$ and $B$ be two SNSs. For any $x \in X$, the following operations are defined:

(1) $A+B=\left\langle T_{A}(x)+T_{B}(x)-\right.$

$T_{A}(x) \cdot T_{B}(x), I_{A}(x)+I_{B}(x)$

$\left.-I_{A}(x) \cdot I_{B}(x), F_{A}(x)+F_{B}(x)-F_{A}(x) \cdot F_{B}(x)\right) ;$

(2) $A \cdot B=\left\langle T_{A}(x) \cdot T_{B}(x), I_{A}(x) \cdot I_{B}(x), F_{A}(x) \cdot F_{B}(x)\right\rangle$;

(3) $\lambda \cdot A=\left\langle 1-\left(1-T_{A}(x)\right)^{\lambda}, 1-\left(1-I_{A}(x)\right)^{\lambda}\right.$,

$\left.1-\left(1-F_{A}(x)\right)^{\lambda}\right), \lambda>0$

(4) $A^{\lambda}=\left\langle T_{A}^{\lambda}(x), I_{A}^{\lambda}(x), F_{A}^{\lambda}(x)\right\rangle, \lambda>0$.
There are some limitations related to Definition 8 and these are now outlined.

(1) In some situations, operations, such as $A+B$ and $A \cdot B$, might be impractical. This can be demonstrated in the example below.

Example 1: Let $A=<0.5,0.5,0.5\rangle$ and $B=$ $<1,0,0>$ be two SNNs. Clearly, $B=<1,0,0>$ is the larger of these SNNs. Theoretically, the sum of any number and the maximum number should be equal to the maximum one. However, according to Definition $8, A+B=<$ $1,0.5,0.5>\neq B$; therefore, the operation ' + ' cannot be accepted. Similar contradictions exist in other operations of Definition 8, and thus those defined above are incorrect.

(1) The correlation coefficient of SNSs (Ye, 2013), which is based on the operations of Definition 8 , cannot be accepted in some special cases.

Example 2: Let $A_{1}=\langle 0.8,0,0\rangle$ and $A_{2}=$ $<0.7,0,0>$ be two SNNs, and $B=<1,0,0>$ be the largest one of the SNNs. According to the correlation coefficient of SNSs (Ye, 2013), $W_{1}\left(A_{1}, B\right)=W_{2}\left(A_{2}, B\right)=1$ can be obtained, but this does not indicate which one is the best. However, it is clear that $A_{1}$ is superior to $A_{2}$.

(1) In addition, the similarity measure for SNSs (Ye, 2014b), which is based on the operations of Definition 8 , cannot be accepted in special cases.

Example 3: Let $A_{1}=\langle 0.1,0,0\rangle$ and $A_{2}=$ $<0.9,0,0>$ be two SNSs, and $B=<1,0,0>$ be the largest one of the SNSs. According to the cosine similarity measure for SNSs (Ye, 2014b), $S_{1}\left(A_{1}, B\right)=S_{2}\left(A_{2}, B\right)=1$ can be obtained, which indicates that $A_{1}$ is equal to $A_{2}$. Yet, it is not possible to discern which one is the best. Since $T_{A_{2}}(x)>T_{A_{1}}(x), I_{A_{2}}(x)>I_{A_{1}}(x)$ and $F_{A_{2}}(x)>F_{A_{1}}(x)$, it is clear that $A_{2}$ is superior to $A_{1}$.

(1) If $I_{A}=I_{B}$, then $A$ and $B$ are both reduced to two intuitionistic fuzzy numbers (IFNs). However, the operations above are not in accordance with the laws of two IFNs (Beliakov, Bustince, Goswami, Mukherjee, \& Pal, 2011; Xu, 2007, 2008, 2010; Yager, 2009).

\section{The operations and comparison method for SNNs}

In this section, the novel operations and comparison method for SNNs are developed based on t-norm and t-conorm. 


\subsection{The operations for SNNs}

According to the operations of IFNs, which are based on the algebraic t-norm and t-conorm (Beliakov et al., 2011; $\mathrm{Xu}, 2007$ ) and Einstein t-norm and t-conorm (Wang \& Liu, 2011, 2012), it can be seen that these operations are all based on different t-norms and t-conorms; therefore, the novel operations of two SNNs can be defined as follows.

Definition 9: Let $A=<T_{A}, I_{A}, F_{A}>$ and $B=$ $<T_{B}, I_{B}, F_{B}>$ be two SNNs, and $\lambda>0$. The operations for SNNs are defined based on the Archimedean t-conorm and t-norm and are as follows:

(1) $\lambda A=<l^{-1}\left(\lambda l\left(T_{A}\right)\right), k^{-1}\left(\lambda k\left(I_{A}\right)\right), k^{-1}\left(\lambda k\left(F_{A}\right)\right)>$;

(2) $A^{\lambda}=<k^{-1}\left(\lambda k\left(T_{A}\right), l^{-1}\left(\lambda l\left(I_{A}\right)\right), l^{-1}\left(\lambda l\left(F_{A}\right)\right)>\right.$;

(3) $A \oplus B=<l^{-1}\left(l\left(T_{A}\right)+l\left(T_{B}\right)\right), k^{-1}\left(k\left(I_{A}\right)+\right.$ $\left.k\left(I_{B}\right)\right), k^{-1}\left(k\left(F_{A}\right)+k\left(F_{B}\right)\right)>$;

(4) $A \otimes B=<k^{-1}\left(k\left(T_{A}\right)+k\left(T_{B}\right)\right), l^{-1}\left(l\left(I_{A}\right)+\right.$ $\left.l\left(I_{B}\right)\right), l^{-1}\left(l\left(F_{A}\right)+l\left(F_{B}\right)\right)>$.

If their generator $k$ is assigned a specific form, then the specific operations of SNNs will be obtained. Let $k(x)=$ $-\log (x)$, therefore

(5) $\lambda A=<1-\left(1-T_{A}\right)^{\lambda},\left(I_{A}\right)^{\lambda},\left(F_{A}\right)^{\lambda}>$;

(6) $A^{\lambda}=<\left(T_{A}\right)^{\lambda}, 1-\left(1-I_{A}\right)^{\lambda}, 1-\left(1-F_{A}\right)^{\lambda}>$;

(7) $A \oplus B=<T_{A}+T_{B}-T_{A} \cdot T_{B}, I_{A} \cdot I_{B}, F_{A}$. $F_{B}>$;

(8) $A \otimes B=<T_{A} \cdot T_{B}, I_{A}+I_{B}-I_{A} \cdot I_{B}, F_{A}+$ $\left.F_{B}-F_{A} \cdot F_{B}\right]>$

Example 4: Based on Example 1 and the operations in Definition 9, let $k(x)=-\log (x), k^{-1}(x)=e^{-x}, l(x)=$ $-\log (1-x)$ and $l^{-1}(x)=1-e^{-x}$, and then the following results are obtained:

$$
\begin{aligned}
A \oplus B & =<0.5+1-0.5 \times 1,0.5 \times 0,0.5 \times 0> \\
& =<1,0,0>=B,
\end{aligned}
$$

which can overcome the drawbacks outlined earlier.

Apparently, SNSs are the extension of IFSs. Let $I_{A}=0, I_{B}=0$ and $T_{A}+F_{A} \leq 1$. Then two SNNs $A=<$ $T_{A}, I_{A}, F_{A}>$ and $B=<T_{B}, I_{B}, F_{B}>$ are reduced to IFNs. According to Definition 9, if $k(x)=-\log (x)$, then the operations coincide with those of IFNs (Beliakov et al., 2011; $\mathrm{Xu}, 2007,2008$, 2010; Yager, 2009). This indicates that the same principles of SNSs in Definition 9 also apply to IFSs. In fact, when the indeterminacy factor $i$ is replaced by $\pi=1-T-F$, the NS becomes an IFS.

Theorem 1: Let $A=<T_{A}, I_{A}, F_{A}>, \quad B=<$ $T_{B}, I_{B}, F_{B}>$ and $C=<T_{C}, I_{C}, F_{C}>$ be three $S N N s$, therefore the following equations are true:

(1) $A \oplus B=B \oplus A$;

(2) $A \otimes B=B \otimes A$;

(3) $\lambda(A \oplus B)=\lambda A \oplus \lambda B, \lambda>0$;

(4) $(A \otimes B)^{\lambda}=A^{\lambda} \otimes B^{\lambda}, \lambda>0$;

(5) $\lambda_{1} A \oplus \lambda_{2} A=\left(\lambda_{1}+\lambda_{2}\right) A, \lambda_{1}>0, \lambda_{2}>0$;

(6) $A^{\lambda_{1}} \otimes A^{\lambda_{2}}=A^{\left(\lambda_{1}+\lambda_{2}\right)}, \lambda_{1}>0, \lambda_{2}>0$;

(7) $(A \oplus B) \oplus C=A \oplus(B \oplus C)$;

(8) $(A \otimes B) \otimes C=A \otimes(B \otimes C)$.

Proof: Equations (1) and (2) are obvious, but the others can also be proved:

$$
\begin{aligned}
& \lambda(A \oplus B) \\
&=\lambda \cdot<l^{-1}\left(l\left(T_{A}\right)+l\left(T_{B}\right)\right), k^{-1}\left(k\left(I_{A}\right)\right. \\
&\left.+k\left(I_{B}\right)\right), k^{-1}\left(k\left(F_{A}\right)+k\left(F_{B}\right)\right)> \\
&=<l^{-1}\left(\lambda l\left(l^{-1}\left(l\left(T_{A}\right)+l\left(T_{B}\right)\right)\right)\right), k^{-1}\left(\lambda k \left(k ^ { - 1 } \left(k\left(I_{A}\right)\right.\right.\right. \\
&\left.\left.\left.+k\left(I_{B}\right)\right)\right)\right), k^{-1}\left(\lambda k\left(k^{-1}\left(k\left(F_{A}\right)+k\left(F_{B}\right)\right)\right)\right)> \\
&=<l^{-1}\left(\lambda\left(l\left(T_{A}\right)+l\left(T_{B}\right)\right)\right), k^{-1}\left(\lambda \left(k\left(I_{A}\right)\right.\right. \\
&\left.\left.+k\left(I_{B}\right)\right)\right), k^{-1}\left(\lambda\left(k\left(F_{A}\right)+k\left(F_{B}\right)\right)\right)> \\
&=\left.<l^{-1}\left(\lambda l\left(T_{A}\right)+\lambda l\left(T_{B}\right)\right)\right), k^{-1}\left(\lambda k\left(I_{A}\right)\right. \\
&\left.\left.\left.+\lambda k\left(I_{B}\right)\right)\right), k^{-1}\left(\lambda k\left(F_{A}\right)+\lambda k\left(F_{B}\right)\right)\right)> \\
&= \lambda A \oplus \lambda B .
\end{aligned}
$$

Therefore, $\lambda(A \oplus B)=\lambda A \oplus \lambda B$.

$$
\begin{aligned}
(A \otimes & B)^{\lambda} \\
= & \left(<k^{-1}\left(k\left(T_{A}\right)+k\left(T_{B}\right)\right), l^{-1}\left(l\left(I_{A}\right)\right.\right. \\
& \left.\left.+l\left(I_{B}\right)\right), l^{-1}\left(l\left(F_{A}\right)+l\left(F_{B}\right)\right)>\right)^{\lambda} \\
= & <k^{-1}\left(\lambda k\left(k^{-1}\left(k\left(T_{A}\right)+k\left(T_{B}\right)\right)\right)\right), l^{-1}\left(\lambda l \left(l ^ { - 1 } \left(l\left(I_{A}\right)\right.\right.\right. \\
& \left.\left.\left.+l\left(I_{B}\right)\right)\right)\right), l^{-1}\left(\lambda l\left(l^{-1}\left(l\left(F_{A}\right)+l\left(F_{B}\right)\right)\right)\right)> \\
= & <k^{-1}\left(\lambda\left(k\left(T_{A}\right)+k\left(T_{B}\right)\right)\right), l^{-1}\left(\lambda \left(l\left(I_{A}\right)\right.\right. \\
& \left.\left.+l\left(I_{B}\right)\right)\right), l^{-1}\left(\lambda\left(l\left(F_{A}\right)+l\left(F_{B}\right)\right)\right)> \\
= & <k^{-1}\left(\lambda k\left(T_{A}\right)+\lambda k\left(T_{B}\right)\right), l^{-1}\left(\lambda l\left(I_{A}\right)\right. \\
& \left.+\lambda l\left(I_{B}\right)\right), l^{-1}\left(\lambda l\left(F_{A}\right)+\lambda l\left(F_{B}\right)\right)>=A^{\lambda} \otimes B^{\lambda} .
\end{aligned}
$$

Therefore, $(A \otimes B)^{\lambda}=A^{\lambda} \otimes B^{\lambda}$.

$$
\begin{aligned}
\lambda_{1} A \oplus & \lambda_{2} A \\
= & <l^{-1}\left(\lambda_{1} l\left(T_{A}\right)\right), k^{-1}\left(\lambda_{1} k\left(I_{A}\right)\right), k^{-1}\left(\lambda_{1} k\left(F_{A}\right)\right)> \\
& \oplus<l^{-1}\left(\lambda_{2} l\left(T_{A}\right)\right), k^{-1}\left(\lambda_{2} k\left(I_{A}\right)\right), \\
& k^{-1}\left(\lambda_{2} k\left(F_{A}\right)\right)>=<l^{-1}\left(l\left(l^{-1}\left(\lambda_{1} l\left(T_{A}\right)\right)\right)\right. \\
& \left.+l\left(l^{-1}\left(\lambda_{2} l\left(T_{A}\right)\right)\right)\right), k^{-1}\left(k\left(k^{-1}\left(\lambda_{1} k\left(I_{A}\right)\right)\right)\right. \\
& \left.+k\left(k^{-1}\left(\lambda_{2} k\left(I_{A}\right)\right)\right)\right) \\
& k^{-1}\left(k\left(k^{-1}\left(\lambda_{1} k\left(F_{A}\right)\right)\right)+k\left(k^{-1}\left(\lambda_{2} k\left(F_{A}\right)\right)\right)\right)> \\
= & <l^{-1}\left(\lambda_{1} l\left(T_{A}\right)+\lambda_{2} l\left(T_{A}\right)\right), k^{-1}\left(\lambda_{1} k\left(I_{A}\right)\right. \\
& \left.+\lambda_{2} k\left(I_{A}\right)\right), k^{-1}\left(\lambda_{1} k\left(F_{A}\right)+\lambda_{2} k\left(F_{A}\right)\right)> \\
= & <l^{-1}\left(\left(\lambda_{1}+\lambda_{2}\right) l\left(T_{A}\right)\right), k^{-1}\left(\left(\lambda_{1}+\lambda_{2}\right) k\left(I_{A}\right)\right), \\
& k^{-1}\left(\left(\lambda_{1}+\lambda_{2}\right) k\left(F_{A}\right)\right)>=\left(\lambda_{1}+\lambda_{2}\right) A .
\end{aligned}
$$


Therefore, $\lambda_{1} A \oplus \lambda_{2} A=\left(\lambda_{1}+\lambda_{2}\right) A$.

$$
\begin{aligned}
A^{\lambda_{1}} \otimes & A^{\lambda_{2}} \\
= & <k^{-1}\left(\lambda_{1} k\left(T_{A}\right), l^{-1}\left(\lambda_{1} l\left(I_{A}\right)\right), l^{-1}\left(\lambda_{1} l\left(F_{A}\right)\right)>\right. \\
& \otimes<k^{-1}\left(\lambda_{2} k\left(T_{A}\right), l^{-1}\left(\lambda_{2} l\left(I_{A}\right)\right), l^{-1}\left(\lambda_{2} l\left(F_{A}\right)\right)>\right. \\
= & <k^{-1}\left(k\left(k^{-1}\left(\lambda_{1} k\left(T_{A}\right)\right)\right)+k\left(k^{-1}\left(\lambda_{2} k\left(T_{A}\right)\right)\right),\right. \\
& l^{-1}\left(l\left(l^{-1}\left(\lambda_{1} l\left(I_{A}\right)\right)\right)+l\left(l^{-1}\left(\lambda_{2} l\left(I_{A}\right)\right)\right),\right. \\
& l^{-1}\left(l\left(l^{-1}\left(\lambda_{1} l\left(F_{A}\right)\right)\right)+l\left(l^{-1}\left(\lambda_{2} l\left(F_{A}\right)\right)\right)>\right. \\
= & <k^{-1}\left(\lambda_{1} k\left(T_{A}\right)+\lambda_{2} k\left(T_{A}\right)\right), l^{-1}\left(\lambda_{1} l\left(I_{A}\right)\right. \\
& \left.+\lambda_{2} l\left(I_{A}\right)\right), l^{-1}\left(\lambda_{1} l\left(F_{A}\right)+\lambda_{2} l\left(F_{A}\right)\right)> \\
= & <k^{-1}\left(\left(\lambda_{1}+\lambda_{2}\right) k\left(T_{A}\right)\right), l^{-1}\left(\left(\lambda_{1}+\lambda_{2}\right) l\left(I_{A}\right)\right), \\
& l^{-1}\left(\left(\lambda_{1}+\lambda_{2}\right) l\left(F_{A}\right)\right)>=A^{\lambda_{1}+\lambda_{2}} .
\end{aligned}
$$

Therefore, $A^{\lambda_{1}} \otimes A^{\lambda_{2}}=A^{\left(\lambda_{1}+\lambda_{2}\right)}$.

$$
\begin{aligned}
(A \oplus & B) \oplus C \\
= & <l^{-1}\left(l\left(T_{A}\right)+l\left(T_{B}\right)\right), k^{-1}\left(k\left(I_{A}\right)\right. \\
& \left.+k\left(I_{B}\right)\right), k^{-1}\left(k\left(F_{A}\right)+k\left(F_{B}\right)\right)> \\
& \oplus<T_{C}, I_{C}, F_{C}> \\
= & <l^{-1}\left(l\left(l^{-1}\left(l\left(T_{A}\right)+l\left(T_{B}\right)\right)\right)\right. \\
& \left.+l\left(T_{C}\right)\right), k^{-1}\left(k\left(k^{-1}\left(k\left(I_{A}\right)+k\left(I_{B}\right)\right)\right)+k\left(I_{C}\right)\right), \\
& k^{-1}\left(k \left(k ^ { - 1 } \left(k\left(F_{A}\right)\right.\right.\right. \\
& \left.\left.\left.+k\left(F_{B}\right)\right)\right)+k\left(F_{C}\right)\right)>=<l^{-1}\left(l\left(T_{A}\right)\right. \\
& \left.+l\left(T_{B}\right)+l\left(T_{C}\right)\right), k^{-1}\left(k\left(I_{A}\right)+k\left(I_{B}\right)\right. \\
& \left.+k\left(I_{C}\right)\right), k^{-1}\left(k\left(F_{A}\right)+k\left(F_{B}\right)+k\left(F_{C}\right)\right)>
\end{aligned}
$$

and

$$
\begin{aligned}
& A \oplus(B \oplus C) \\
& =<T_{A}, I_{A}, F_{A}>\oplus<l^{-1}\left(l\left(T_{B}\right)+l\left(T_{C}\right)\right), \\
& k^{-1}\left(k\left(I_{B}\right)+k\left(I_{C}\right)\right), k^{-1}\left(k\left(F_{B}\right)+k\left(F_{C}\right)\right)> \\
& =l^{-1}\left(l\left(T_{A}\right)+l\left(l^{-1}\left(l\left(T_{B}\right)+l\left(T_{C}\right)\right)\right)\right) \text {, } \\
& k^{-1}\left(k\left(I_{A}\right)+k\left(k^{-1}\left(k\left(I_{B}\right)+k\left(I_{C}\right)\right)\right)\right), k^{-1}\left(k\left(F_{A}\right)\right. \\
& \left.+k\left(k^{-1}\left(k\left(F_{B}\right)+k\left(F_{C}\right)\right)\right)\right)> \\
& =<l^{-1}\left(l\left(T_{A}\right)+l\left(T_{B}\right)+l\left(T_{C}\right)\right), k^{-1}\left(k\left(I_{A}\right)\right. \\
& \left.+k\left(I_{B}\right)+k\left(I_{C}\right)\right), k^{-1}\left(k\left(F_{A}\right)\right. \\
& \left.+k\left(F_{B}\right)+k\left(F_{C}\right)\right)>\text {. }
\end{aligned}
$$

Therefore, $(A \oplus B) \oplus C=A \oplus(B \oplus C)$.

$$
\begin{aligned}
(A \otimes & B) \otimes C \\
= & <k^{-1}\left(k\left(T_{A}\right)+k\left(T_{B}\right)\right), l^{-1}\left(l\left(I_{A}\right)+l\left(I_{B}\right)\right), \\
& l^{-1}\left(l\left(F_{A}\right)+l\left(F_{B}\right)\right)>\otimes<T_{C}, I_{C}, F_{C}> \\
= & \left.\left.<k^{-1}\left(k\left(k^{-1}\left(k\left(T_{A}\right)+k\left(T_{B}\right)\right)\right)+k\left(T_{C}\right)\right)\right)\right), \\
& \left.\left.l^{-1}\left(l\left(l^{-1}\left(l\left(I_{A}\right)+l\left(I_{B}\right)\right)\right)+l\left(I_{C}\right)\right)\right)\right), \\
& \left.\left.l^{-1}\left(l\left(l^{-1}\left(l\left(I_{A}\right)+l\left(I_{B}\right)\right)\right)+l\left(I_{C}\right)\right)\right)\right)> \\
= & <k^{-1}\left(k\left(T_{A}\right)+k\left(T_{B}\right)+k\left(T_{C}\right)\right), l^{-1}\left(l\left(I_{A}\right)\right. \\
& \left.+l\left(I_{B}\right)+l\left(I_{C}\right)\right), l^{-1}\left(l\left(I_{A}\right)+l\left(I_{B}\right)+l\left(I_{C}\right)\right)>
\end{aligned}
$$

and

$$
\begin{aligned}
& A \otimes(B \otimes C) \\
& =<T_{A}, I_{A}, F_{A}>\otimes<k^{-1}\left(k\left(T_{B}\right)+k\left(T_{C}\right)\right), \\
& l^{-1}\left(l\left(I_{B}\right)+l\left(I_{C}\right)\right), l^{-1}\left(l\left(F_{B}\right)+l\left(F_{C}\right)\right)>
\end{aligned}
$$

$$
\begin{aligned}
= & k^{-1}\left(k\left(T_{A}\right)+k\left(k^{-1}\left(k\left(T_{B}\right)+k\left(T_{C}\right)\right)\right)\right), l^{-1}\left(l\left(I_{A}\right)\right. \\
& \left.+l\left(l^{-1}\left(l\left(I_{B}\right)+l\left(I_{C}\right)\right)\right)\right), l^{-1}\left(l\left(F_{A}\right)+l\left(l ^ { - 1 } \left(l\left(F_{B}\right)\right.\right.\right. \\
& \left.\left.\left.+l\left(F_{C}\right)\right)\right)\right)> \\
= & <k^{-1}\left(k\left(T_{A}\right)+k\left(T_{B}\right)+k\left(T_{C}\right)\right), l^{-1}\left(l\left(I_{A}\right)\right. \\
& \left.+l\left(I_{B}\right)+l\left(I_{C}\right)\right), l^{-1}\left(l\left(I_{A}\right)+l\left(I_{B}\right)+l\left(I_{C}\right)\right)>.
\end{aligned}
$$

Therefore, $(A \otimes B) \otimes C=A \otimes(B \otimes C)$.

The proof is therefore complete.

\subsection{The comparison method of SNNS}

Based on the score function and accuracy function of IFNs (Xu, 2007, 2008, 2010; Yager, 2009), the score function, accuracy function and certainty function of an $\mathrm{SNN}$ are defined as follows.

Definition 10: Let $A=<T_{A}, I_{A}, F_{A}>$ be an SNN, and then the score function $s(A)$, accuracy function $a(A)$ and certainty function $c(A)$ of an $\mathrm{SNN}$ are defined as follows:

(1) $s(A)=\left(T_{A}+1-I_{A}+1-F_{A}\right) / 3$;

(2) $a(A)=T_{A}-F_{A}$;

(3) $c(A)=T_{A}$.

The score function is an important index in ranking SNNs. For an SNN $A$, the bigger the truth-membership $T_{A}$ is, the greater the SNN will be; furthermore, the smaller the indeterminacy-membership $I_{A}$ is, the greater the SNN will be; similarly, the smaller the false-membership $F_{A}$ is, the greater the SNN will be. For the accuracy function, the bigger the difference between truth and falsity, the more affirmative the statement is. As for the certainty function, the certainty of any SNN positively depends on the value of truth- membership $T_{A}$.

On the basis of Definition 10, the method for comparing SNNs can be defined as follows.

Definition 11: Let $A$ and $B$ be two SNNs. The comparison method can be defined as follows:

(1) if $s(A)>s(B)$, then $A$ is greater than $B$, that is, $A$ is superior to $B$, denoted by $A \succ B$;

(2) if $s(A)=s(B)$ and $a(A)>a(B)$, then $A$ is greater than $B$, that is, $A$ is superior to $B$, denoted by $A \succ B$;

(3) if $s(A)=s(B), a(A)=a(B)$ and $c(A)>c(B)$, then $A$ is greater than $B$, that is, $A$ is superior to $B$, denoted by $A \succ B$;

(4) if $s(A)=s(B), a(A)=a(B)$ and $c(A)=c(B)$, then $A$ is equal to $B$, that is, $A$ is indifferent to $B$, denoted by $A \sim B$.

Example 5: Based on Example 2 and Definition 10, $s\left(A_{1}\right)=\frac{0.8+1-0+1-0}{3}=\frac{2.8}{3}$ and $s\left(A_{2}\right)=\frac{0.7+1-0+1-0}{3}=\frac{2.7}{3}$ can be obtained. According to Definition 11, $s\left(A_{1}\right)>$ $s\left(A_{2}\right)$; therefore, $A_{1} \succ A_{2}$, i.e., $A_{1}$ is superior $A_{2}$, which avoids the drawbacks discussed in Example 2. 
Example 6: Based on Example 3 and Definition 10, $s\left(A_{1}\right)<s\left(A_{2}\right)$, then $A_{2} \succ A_{1}$, i.e., $A_{2}$ is superior to $A_{1}$, which also avoids the shortcomings discussed in Example 3.

\section{The aggregation operators of SNNs and their applications to MCGDM problems}

In this section, by applying the SNNs' operations, the aggregation operators of SNNs are presented and a method for MCGDM problems that utilises the proposed aggregation operators is proposed.

\subsection{The aggregation operators of SNNs}

Definition 12: Let $A_{j}=<T_{A_{j}}, I_{A_{j}}, F_{A_{j}}>(j=1$, $2, \ldots, n)$ be a collection of SNNs, SNNWA : $\mathrm{SNN}^{n} \rightarrow$ $\mathrm{SNN}$,

$$
\operatorname{SNNWA}_{w}\left(A_{1}, A_{2}, \ldots, A_{n}\right)=\sum_{j=1}^{n} w_{j} A_{j}
$$

the SNNWA operator is called the simplified neutrosophic number weighted averaging operator of dimension $n$, where $w=\left(w_{1}, w_{2}, \ldots, w_{n}\right)$ is the weight vector of $A_{j}(j=1,2, \ldots, n)$, with $w_{j} \geq 0(j=1,2, \ldots, n)$ and $\sum_{j=1}^{n} w_{j}=1$.

Theorem 2: Let $A_{j}=<T_{A_{j}}, I_{A_{j}}, F_{A_{j}}>\quad(j=1$, $2, \ldots, n)$ be a collection of SNNs, and $w=$ $\left(w_{1}, w_{2}, \ldots, w_{n}\right)$ be the weight vector of $A_{j}$ $(j=1,2, \ldots, n)$, with $w_{j} \geq 0 \quad(j=1,2, \ldots, n)$ and $\sum_{j=1}^{n} w_{j}=1$.Then their aggregated result using the SNNWA operator is also an SNN, and

$$
\begin{gathered}
\operatorname{SNNWA}_{w}\left(A_{1}, A_{2}, \ldots, A_{n}\right)=\left\langle l^{-1}\left(\sum_{j=1}^{n} w_{j} l\left(T_{A_{j}}\right)\right),\right. \\
\left.k^{-1}\left(\sum_{j=1}^{n} w_{j} k\left(I_{A_{j}}\right)\right), k^{-1}\left(\sum_{j=1}^{n} w_{j} k\left(F_{A_{j}}\right)\right)\right\rangle
\end{gathered}
$$

where $k$ is the additive generator of the Archimedean t-norm that is used in the operations of SNNs and $l(x)=k(1-x)$.

If $k(x)=-\log (x)$, then $l(x)=-\log (1-x), k^{-1}(x)=$ $e^{-x}$ and $l^{-1}(x)=1-e^{-x}$. Thus, the aggregated result using the SNNWA operator in Theorem 2 can be represented by

$$
\begin{aligned}
& \operatorname{SNNWA}_{w}\left(A_{1}, A_{2}, \ldots, A_{n}\right)=\left\langle 1-\prod_{j=1}^{n}\left(1-T_{A_{j}}\right)^{w_{j}},\right. \\
& \left.\prod_{j=1}^{n} I_{A_{j}}^{w_{j}}, \prod_{j=1}^{n} F_{A_{j}}^{w_{j}}\right\rangle
\end{aligned}
$$

If $k(x)=\log \left(\frac{2-x}{x}\right)$, then Theorem 2 can be represented by

$$
\begin{aligned}
& \operatorname{SNNWA}_{w}\left(A_{1}, A_{2}, \ldots, A_{n}\right) \\
& =\left\langle\frac{\prod_{j=1}^{n}\left(1+T_{A_{j}}\right)^{w_{j}}-\prod_{j=1}^{n}\left(1-T_{A_{j}}\right)^{w_{j}}}{\prod_{j=1}^{n}\left(1+T_{A_{j}}\right)^{w_{j}}+\prod_{j=1}^{n}\left(1-T_{A_{j}}\right)^{w_{j}}},\right. \\
& \quad \times \frac{2 \prod_{j=1}^{n} I_{A_{j}}^{w_{j}}}{\prod_{j=1}^{n}\left(2-I_{A_{j}}\right)^{w_{j}}+\prod_{j=1}^{n} I_{A_{j}}^{w_{j}}} \\
& \left.\quad \times \frac{2 \prod_{i=1}^{n} F_{A_{j}}^{w_{j}}}{\prod_{j=1}^{n}\left(2-F_{A_{j}}\right)^{w_{j}}+\prod_{j=1}^{n} F_{A_{j}}^{w_{j}}}\right\rangle .
\end{aligned}
$$

Proof: By using the mathematical induction on $n$ :

(1) let $n=2$, since

$$
\begin{aligned}
& w_{1} A_{1} \oplus w_{2} A_{2} \\
&=<l^{-1}\left(w_{1} l\left(T_{A_{1}}\right)\right), k^{-1}\left(w_{1} k\left(I_{A_{1}}\right)\right), k^{-1}\left(w_{1} k\left(F_{A_{1}}\right)\right)> \\
& \oplus<l^{-1}\left(w_{2} l\left(T_{A_{2}}\right)\right), k^{-1}\left(w_{2} k\left(I_{A_{2}}\right)\right), k^{-1}\left(w_{2} k\left(F_{A_{2}}\right)\right)> \\
&=<l^{-1}\left(l \left(l^{-1}\left(w_{1} l\left(T_{A_{1}}\right)\right)+l\left(l^{-1}\left(w_{2} l\left(T_{A_{2}}\right)\right)\right)\right.\right. \\
& k^{-1}\left(k \left(k^{-1}\left(w_{1} k\left(I_{A_{1}}\right)\right)+k\left(k^{-1}\left(w_{2} k\left(I_{A_{2}}\right)\right)\right)\right.\right. \\
& k^{-1}\left(k \left(k^{-1}\left(w_{1} k\left(F_{A_{1}}\right)\right)+k\left(k^{-1}\left(w_{2} k\left(F_{A_{2}}\right)\right)\right)\right.\right. \\
&=<l^{-1}\left(w_{1} l\left(T_{A_{1}}\right)+w_{2} l\left(T_{A_{2}}\right)\right), k^{-1}\left(w_{1} k\left(I_{A_{1}}\right)\right. \\
&\left.+w_{2} k\left(I_{A_{2}}\right)\right), k^{-1}\left(w_{1} k\left(F_{A_{1}}\right)+w_{2} k\left(F_{A_{2}}\right)\right)>
\end{aligned}
$$

then

$$
\begin{aligned}
& \operatorname{SNNWA}_{w}\left(A_{1}, A_{2}\right)=w_{1} A_{1} \oplus w_{2} A_{2} \\
&=<l^{-1}\left(w_{1} l\left(T_{A_{1}}\right)+w_{2} l\left(T_{A_{2}}\right)\right), k^{-1}\left(w_{1} k\left(I_{A_{1}}\right)\right. \\
&\left.+w_{2} k\left(I_{A_{2}}\right)\right), k^{-1}\left(w_{1} k\left(F_{A_{1}}\right)+w_{2} k\left(F_{A_{2}}\right)\right)>;
\end{aligned}
$$

(1) if Equation (3) holds for $n=k$, then

$$
\begin{aligned}
\operatorname{SNNWA}_{w}\left(A_{1}, A_{2}, \ldots, A_{k}\right)=w_{1} A_{1} \oplus w_{2} A_{2} \oplus \cdots \oplus w_{k} A_{k} \\
=\left\langle l^{-1}\left(\sum_{j=1}^{k} w_{j} l\left(T_{A_{j}}\right)\right), k^{-1}\left(\sum_{j=1}^{k} w_{j} k\left(I_{A_{j}}\right)\right),\right. \\
\left.k^{-1}\left(\sum_{j=1}^{k} w_{j} k\left(F_{A_{j}}\right)\right)\right\rangle .
\end{aligned}
$$

If $n=k+1$, by operations (1) and (3) in Definition 8 $\operatorname{SNNWA}_{w}\left(A_{1}, A_{2}, \ldots, A_{k}, A_{k+1}\right)$

$$
\begin{aligned}
&=\left(w_{1} A_{1} \oplus w_{2} A_{2} \oplus \cdots \oplus w_{k} A_{k}\right) \oplus w_{k+1} A_{k+1} \\
&=\left\langle l^{-1}\left(\sum_{i=1}^{k} w_{i} l\left(T_{A_{i}}\right)\right), k^{-1}\left(\sum_{i=1}^{k} w_{i} k\left(I_{A_{i}}\right)\right),\right. \\
&\left.k^{-1}\left(\sum_{i=1}^{k} w_{i} k\left(F_{A_{i}}\right)\right)\right\rangle \\
& \oplus\left\langle l^{-1}\left(w_{k+1} l\left(T_{A_{k+1}}\right)\right), k^{-1}\left(w_{k+1} k\left(I_{A_{k+1}}\right)\right),\right. \\
&\left.k^{-1}\left(w_{k+1} k\left(F_{A_{k+1}}\right)\right)\right\rangle
\end{aligned}
$$




$$
\begin{aligned}
= & \left\langle l^{-1}\left(l\left(l^{-1}\left(\sum_{j=1}^{k} w_{j} l\left(T_{A_{j}}\right)\right)+l\left(l^{-1}\left(w_{k+1} l\left(T_{A_{k+1}}\right)\right)\right)\right)\right),\right. \\
& k^{-1}\left(k\left(k^{-1}\left(\sum_{j=1}^{k} w_{j} k\left(I_{A_{j}}\right)\right)+k\left(k^{-1}\left(w_{k+1} k\left(I_{A_{k+1}}\right)\right)\right)\right)\right), \\
& \left.k^{-1}\left(k\left(k^{-1}\left(\sum_{j=1}^{k} w_{j} k\left(F_{A_{j}}\right)\right)\right)+k\left(k^{-1}\left(w_{k+1} k\left(F_{A_{k+1}}\right)\right)\right)\right)\right\rangle \\
= & \left\langle l^{-1}\left(\sum_{j=1}^{k+1} w_{j} l\left(T_{A_{j}}\right)\right), k^{-1}\left(\sum_{j=1}^{k+1} w_{j} k\left(I_{A_{j}}\right)\right),\right. \\
& \left.k^{-1}\left(\sum_{j=1}^{k+1} w_{j} k\left(F_{A_{j}}\right)\right)\right\rangle,
\end{aligned}
$$

i.e., Equation (2) holds for $n=k+1$. Thus, Equation (2) holds for all $n$. Then

$$
\begin{gathered}
\operatorname{SNNWA}_{w}\left(A_{1}, A_{2}, \ldots, A_{n}\right)=\left\langle l^{-1}\left(\sum_{j=1}^{n} w_{j} l\left(T_{A_{j}}\right)\right),\right. \\
\left.k^{-1}\left(\sum_{j=1}^{n} w_{j} k\left(I_{A_{j}}\right)\right), k^{-1}\left(\sum_{j=1}^{n} w_{j} k\left(F_{A_{j}}\right)\right)\right\rangle .
\end{gathered}
$$

The proof is therefore complete.

It is clear that the SNNWA operator has the following properties.

Property 1: Let $A_{j}=<T_{A_{j}}, I_{A_{j}}, F_{A_{j}}>(j=1$, $2, \ldots, n)$ be a collection of SNNs.

(1) Idempotency: if all $A_{j}(j=1,2, \ldots, n)$ are equal, i.e., $A_{j}=A$, for all $j \in\{1,2, \ldots, n\}$, then $\operatorname{SNNWA}_{w}\left(A_{1}, A_{2}, \ldots, A_{n}\right)=A$.

(2) Boundedness: assume $A^{-}=<\min _{j} T_{A_{j}}$, $\max _{j} I_{A_{j}}$, $\max _{j} F_{A_{j}}>\quad$ and $\quad A^{+}=<\max _{j} T_{A_{j}}, \min _{j} I_{A_{j}}$, $\min _{j} F_{A_{j}}>$ for all $j \in\{1,2, \ldots, n\}$, and then $A^{-} \subseteq \operatorname{SNNWA}_{w}\left(A_{1}, A_{2}, \ldots, A_{n}\right) \subseteq A^{+}$.

(3) Monotonity: assume $A_{j}^{*}=<T_{A_{j}^{*}}, I_{A_{j}^{*}}, F_{A_{j}^{*}}>$ and $A_{j} \subseteq A_{j}^{*} \quad$ for any $j \in\{1,2, \ldots, n\}$, and then $\operatorname{SNNWA}_{w}\left(A_{1}, A_{2}, \ldots, A_{n}\right)$ $\subseteq \operatorname{SNNWA}_{w}\left(A_{1}^{*}, A_{2}^{*}, \ldots, A_{n}^{*}\right)$.

Proof: Let $A_{j}=<T_{A_{j}}, I_{A_{j}}, F_{A_{j}}>(j=1,2, \ldots, n)$ be a collection of SNNs, $w=\left(w_{1}, w_{2}, \ldots, w_{n}\right)$ be the weight vector of $A_{j}(j=1,2, \ldots, n)$, with $w_{j} \geq 0(j=$ $1,2, \ldots, n)$ and $\sum_{j=1}^{n} w_{j}=1$.

(1) Since $A_{1}=A_{2}=\cdots=A_{n}=A=<T_{A}, I_{A}$, $F_{A}>$ and $\sum_{j=1}^{n} w_{j}=1$,

$$
\begin{aligned}
\operatorname{SNNWA}_{w}\left(A_{1}, A_{2}, \ldots, A_{n}\right) \\
=\left\langle l^{-1}\left(\sum_{j=1}^{n} w_{j} l\left(T_{A}\right)\right),\right. \\
\left.k^{-1}\left(\sum_{j=1}^{n} w_{j} k\left(I_{A}\right)\right), k^{-1}\left(\sum_{j=1}^{n} w_{j} k\left(F_{A}\right)\right)\right\rangle \\
=\left\langle l^{-1}\left(\sum_{j=1}^{n} w_{j} l\left(T_{A}\right)\right), k^{-1}\left(\sum_{j=1}^{n} w_{j} k\left(I_{A}\right)\right),\right. \\
=<l^{-1}\left(l\left(\sum_{j=1}^{n} w_{j} k\left(F_{A}\right)\right), k^{-1}\left(k\left(I_{A}\right)\right), k^{-1}\left(k\left(F_{A}\right)\right)>\right. \\
=<T_{A}, I_{A}, F_{A}>.
\end{aligned}
$$

(2) For any $A_{j}=<T_{A_{j}}, I_{A_{j}}, F_{A_{j}}>\quad(1 \leq$ $j \leq n), \quad$ it is clear that $A^{-}=<$ $\min _{j} T_{A_{j}}, \max _{j} I_{A_{j}}, \max _{j} F_{A_{j}}>$ and $A^{+}=$ $<\max _{j} T_{A_{j}}, \min _{j} I_{A_{j}}, \min _{j} F_{A_{j}}>$ are two SNNs. For any $j, \min _{j} T_{A_{j}} \leq T_{A_{j}} \leq \max _{j} T_{A_{j}}$, $\min _{j} I_{A_{j}} \leq I_{A_{j}} \leq \max _{j} I_{A_{j}} \quad$ and $\min _{j} F_{A_{j}} \leq$ $F_{A_{j}} \leq \max _{j} F_{A_{j}} . \quad$ Since $\quad \sum_{j=1}^{n} w_{j}=1$, $l(x)=k(1-x)$, and $l(x)$ is a strictly increasing function, then

$$
\begin{aligned}
& \max _{j} T_{A_{j}}=l^{-1}\left(\sum_{j=1}^{n} w_{j} l\left(\max _{j} T_{A_{j}}\right)\right) \\
& \leq l^{-1}\left(\sum_{j=1}^{n} w_{j} l\left(T_{A_{j}}\right)\right) \leq l^{-1}\left(\sum_{j=1}^{n} w_{j} l\left(\min _{j} T_{A_{j}}\right)\right) \\
& =\min _{j} T_{A_{j}} ;
\end{aligned}
$$

$$
\begin{aligned}
& \min _{j} I_{A_{j}}=k^{-1}\left(\sum_{j=1}^{n} w_{j} k\left(\min _{j} I_{A_{j}}\right)\right) \\
& \leq k^{-1}\left(\sum_{j=1}^{n} w_{j} k\left(I_{A_{j}}\right)\right) \leq k^{-1}\left(\sum_{j=1}^{n} w_{j} k\left(\max _{j} I_{A_{j}}\right)\right) \\
& =\max _{j} I_{A_{j}} ;
\end{aligned}
$$

$$
\begin{aligned}
& \min _{j} F_{A_{j}}=k^{-1}\left(\sum_{j=1}^{n} w_{j} k\left(\min _{j} F_{A_{j}}\right)\right) \\
& \leq k^{-1}\left(\sum_{j=1}^{n} w_{j} k\left(F_{A_{j}}\right)\right) \leq k^{-1}\left(\sum_{j=1}^{n} w_{j} k\left(\max _{j} F_{A_{j}}\right)\right) \\
& =\max _{j} F_{A_{j}} .
\end{aligned}
$$


Based on Definition 7, $A^{-} \subseteq$ $\operatorname{SNNWA}_{w}\left(A_{1}, A_{2}, \ldots, A_{n}\right) \subseteq A^{+}$.

(3) For $A_{j}=<T_{A_{j}}, I_{A_{j}}, F_{A_{j}}>$ and $A_{j}^{*}=<$ $T_{A_{j}^{*}}, I_{A_{j}^{*}}, F_{A_{j}^{*}}>$. Since $A_{j} \subseteq A_{j}^{*}$, therefore $T_{A_{j}} \leq T_{A_{i}^{*}}, I_{A_{j}} \geq I_{A_{j}^{*}}$ and $F_{A_{j}} \geq F_{A_{i}^{*}}$. Because $l(x)=k(1-x)$, and $l(x)$ is a strictly increasing function, therefore $l^{-1}\left(\sum_{j=1}^{n} w_{j} l\left(T_{A_{j}}\right)\right) \leq$ $l^{-1}\left(\sum_{j=1}^{n} w_{j} l\left(T_{A_{j}^{*}}\right)\right), \quad k^{-1}\left(\sum_{j=1}^{n} w_{j} k\left(I_{A_{j}}\right)\right) \geq$ $k^{-1}\left(\sum_{j=1}^{n} w_{j} k\left(I_{A_{j}^{*}}\right)\right)$ and $k^{-1}\left(\sum_{j=1}^{n} w_{j} k\left(F_{A_{j}}\right)\right)$ $\geq k^{-1}\left(\sum_{j=1}^{n} w_{j} k\left(F_{A_{j}^{*}}\right)\right)$. According to Definition $7, \quad \operatorname{SNNWA}_{w}\left(A_{1}, A_{2}, \ldots, A_{n}\right) \subseteq$ $\operatorname{SNNWA}_{w}\left(A_{1}^{*}, A_{2}^{*}, \ldots, A_{n}^{*}\right)$.

The proof is therefore complete.

Definition 13: Let $A_{j}=<T_{A_{j}}, I_{A_{j}}, F_{A_{j}}>\quad(j=$ $1,2, \ldots, n)$ be a collection of SNNs, SNNWG : $\mathrm{SNN}^{n} \rightarrow$ SNN,

$$
\operatorname{SNNWG}_{w}\left(A_{1}, A_{2}, \ldots, A_{n}\right)=\prod_{j=1}^{n} A_{j}^{w_{j}},
$$

and thus the SNNWG operator is called a simplified neutrosophic number weighted geometric operator of dimension $n$, where $w=\left(w_{1}, w_{2}, \ldots, w_{n}\right)$ is the weight vector of $A_{j}(j=1,2, \ldots, n)$, with $w_{j} \geq 0(j=1,2, \ldots, n)$ and $\sum_{j=1}^{n} w_{j}=1$.

Theorem 3: Let $A_{j}=<T_{A_{j}}, I_{A_{j}}, F_{A_{j}}>\quad(j=$ $1,2, \ldots, n)$ be a collection of $S N N s$, and $w=$ $\left(w_{1}, w_{2}, \ldots, w_{n}\right)$ be the vector of $A_{j}(j=1,2, \ldots, n)$, with $w_{j} \in[0,1]$ and $\sum_{j=1}^{n} w_{j}=1$. Then their aggregated result using the $S N N W G$ operator is also an $S N N$, and

$$
\begin{gathered}
\operatorname{SNNWG}_{w}\left(A_{1}, A_{2}, \ldots, A_{n}\right)=\left\langle k^{-1}\left(\sum_{j=1}^{n} w_{j} k\left(T_{A_{j}}\right)\right),\right. \\
\left.l^{-1}\left(\sum_{j=1}^{n} w_{j} l\left(I_{A_{j}}\right)\right), l^{-1}\left(\sum_{j=1}^{n} w_{j} l\left(F_{A_{j}}\right)\right)\right\rangle .
\end{gathered}
$$

Let $k(x)=-\log (x)$, and then Theorem 3 can be represented by

$$
\begin{aligned}
& \operatorname{SNNWG}_{w}\left(A_{1}, A_{2}, \ldots, A_{n}\right)=\left\langle\prod_{j=1}^{n} T_{A_{j}}^{w_{j}},\right. \\
& \left.1-\prod_{j=1}^{n}\left(1-I_{A_{j}}\right)^{w_{j}}, 1-\prod_{j=1}^{n}\left(1-F_{A_{j}}\right)^{w_{j}}\right\rangle .
\end{aligned}
$$
by

Let $k(x)=\log \left(\frac{2-x}{x}\right)$, and Theorem 3 can be denoted

$$
\begin{aligned}
& \operatorname{SNNWG}_{w}\left(A_{1}, A_{2}, \ldots, A_{n}\right) \\
& =\left\langle\frac{2 \prod_{j=1}^{n} T_{A_{j}}^{w_{j}}}{\prod_{j=1}^{n}\left(2-T_{A_{j}}\right)^{w_{j}}+\prod_{j=1}^{n} T_{A_{j}}^{w_{j}}},\right. \\
& \times \frac{\prod_{j=1}^{n}\left(1+I_{A_{j}}\right)^{w_{j}}-\prod_{j=1}^{n}\left(1-I_{A_{j}}\right)^{w_{j}}}{\prod_{j=1}^{n}\left(1+I_{A_{j}}\right)^{w_{j}}+\prod_{j=1}^{n}\left(1-I_{A_{j}}\right)^{w_{j}}}, \\
& \left.\times \frac{\prod_{j=1}^{n}\left(1+F_{A_{j}}\right)^{w_{j}}-\prod_{j=1}^{n}\left(1-F_{A_{j}}\right)^{w_{j}}}{\prod_{j=1}^{n}\left(1+F_{A_{j}}\right)^{w_{j}}+\prod_{j=1}^{n}\left(1-F_{A_{j}}\right)^{w_{j}}}\right\rangle .
\end{aligned}
$$

Proof: Theorem 3 can be proved by the mathematical induction method, and the process is omitted here.

Property 2: Let $A_{j}=<T_{A_{j}}, I_{A_{j}}, F_{A_{j}}>(j=$ $1,2, \ldots, n)$ be a collection of SNNs.

(1) Idempotency: if all $A_{j}(j=1,2, \ldots, n)$ are equal, i.e., $A_{j}=A$, for all $j \in\{1,2, \ldots, n\}$, then $\mathrm{SNNWG}_{w}\left(A_{1}, A_{2}, \ldots, A_{n}\right)=A$.

(2) Boundedness: assume $A^{-}=<\min _{j} T_{A_{j}}, \max _{j} I_{A_{j}}$, $\max _{j} F_{A_{j}}>\quad$ and $\quad A^{+}=<\max _{j} T_{A_{j}}, \min _{j} I_{A_{j}}$, $\min _{j} F_{A_{j}}>$ for all $j \in\{1,2, \ldots, n\}$, and then $A^{-} \subseteq \operatorname{SNNWG}_{w}\left(A_{1}, A_{2}, \ldots, A_{n}\right) \subseteq A^{+}$.

(3) Monotonity: assume $A_{j}^{*}=<T_{A_{j}^{*}}, I_{A_{j}^{*}}, F_{A_{j}^{*}}>$ and $A_{j} \subseteq A_{j}^{*} \quad$ for any $j \in\{1,2, \ldots, n\}$, and then $\operatorname{SNNWG}_{w}\left(A_{1}, A_{2}, \ldots, A_{n}\right)$ $\subseteq \operatorname{SNNWG}_{w}\left(A_{1}^{*}, A_{2}^{*}, \ldots, A_{n}^{*}\right)$.

Proof: The proof is similar to that for Property 1 .

Definition 14: Let $A_{j}=<T_{A_{j}}, I_{A_{j}}, F_{A_{j}}>\quad(j=$ $1,2, \ldots, n)$ be a collection of SNNs, SNNOWA : $\mathrm{SNN}^{n} \rightarrow$ $\mathrm{SNN}$,

$$
\operatorname{SNNOWA}_{\omega}\left(A_{1}, A_{2}, \ldots, A_{n}\right)=\sum_{j=1}^{n} \omega_{j} A_{\sigma(j)},
$$

and thus the SNNOWA operator is called the simplified neutrosophic number ordered weighted averaging operator of dimension $n$, where $A_{\sigma(j)}$ is the $j$ th largest value. $\omega=$ $\left(\omega_{1}, \omega_{2}, \ldots, \omega_{n}\right)$ is the aggregation-associated vector such that $\omega_{j} \geq 0(j=1,2, \ldots, n)$ and $\sum_{j=1}^{n} \omega_{j}=1$.

Theorem 4: Let $A_{j}=<T_{A_{j}}, I_{A_{j}}, F_{A_{j}}>\quad(j=$ $1,2, \ldots, n)$ be a collection of SNNs, and $\omega=\left(\omega_{1}, \omega_{2}, \ldots, \omega_{n}\right)$ be the aggregation-associated vector such that $\omega_{j} \geq 0(j=1,2, \ldots, n)$ and $\sum_{j=1}^{n} \omega_{j}=1$. Their aggregated result using the SNNOWA operator is also an SNN, and 


$$
\begin{aligned}
& \operatorname{SNNOWA}_{\omega}\left(A_{1}, A_{2}, \ldots, A_{n}\right) \\
& =\left\langle l^{-1}\left(\sum_{j=1}^{n} \omega_{j} l\left(T_{A_{\sigma(j)}}\right)\right)\right. \\
& \left.k^{-1}\left(\sum_{j=1}^{n} \omega_{j} k\left(I_{A_{\sigma(j)}}\right)\right), k^{-1}\left(\sum_{j=1}^{n} \omega_{j} k\left(F_{A_{\sigma(j)}}\right)\right)\right\rangle .
\end{aligned}
$$

Let $k(x)=-\log (x)$, and then Theorem 4 can be represented by

$$
\begin{aligned}
& \operatorname{SNNOWA}_{\omega}\left(A_{1}, A_{2}, \ldots, A_{n}\right) \\
& \quad=\left\langle 1-\prod_{j=1}^{n}\left(1-T_{A_{\sigma(j)}}\right)^{\omega_{j}}, \prod_{j=1}^{n} I_{A_{\sigma(j)}}^{\omega_{j}}, \prod_{j=1}^{n} F_{A_{\sigma(j)}}^{\omega_{j}}\right\rangle .
\end{aligned}
$$
by

If $k(x)=\log \left(\frac{2-x}{x}\right)$, then Theorem 4 can be represented

$$
\begin{aligned}
& \operatorname{SNNOWA}_{\omega}\left(A_{1}, A_{2}, \ldots, A_{n}\right) \\
& =\left\langle\frac{\prod_{j=1}^{n}\left(1+T_{A_{\sigma(j)}}\right)^{\omega_{j}}-\prod_{j=1}^{n}\left(1-T_{A_{\sigma(j)}}\right)^{\omega_{j}}}{\prod_{j=1}^{n}\left(1+T_{A_{\sigma(j)}}\right)^{\omega_{j}}+\prod_{j=1}^{n}\left(1-T_{A_{\sigma(j)}}\right)^{\omega_{j}}},\right. \\
& \quad \times \frac{2 \prod_{j=1}^{n} I_{A_{\sigma(j)}}^{\omega_{j}}}{\prod_{j=1}^{n}\left(2-I_{A_{\sigma(j)}}\right)^{\omega_{j}}+\prod_{j=1}^{n} I_{A_{\sigma(j)}}^{\omega_{j}}} \\
& \left.\quad \times \frac{2 \prod_{j=1}^{n} F_{A_{\sigma(j)}}^{\omega_{j}}}{\prod_{j=1}^{n}\left(2-F_{A_{\sigma(j)}}\right)^{\omega_{j}}+\prod_{j=1}^{n} F_{A_{\sigma(j)}}^{\omega_{j}}}\right\rangle,
\end{aligned}
$$

where $A_{\sigma(j)}$ is the $j$ th largest value according to the total order $A_{\sigma(1)} \geq A_{\sigma(2)} \geq \cdots \geq A_{\sigma(n)}$.

Proof: Theorem 4 can be proved by the mathematical induction method, and the process is omitted here.

Property 3: Let $A_{j}=<T_{A_{j}}, I_{A_{j}}, F_{A_{j}}>(j=1,2, \ldots, n)$ be a collection of SNNs.

(1) Idempotency: if all $A_{j}(j=1,2, \ldots, n)$ are equal, i.e., $A_{j}=A$, for all $j \in\{1,2, \ldots, n\}$, then $\operatorname{SNNOWA}_{\omega}\left(A_{1}, A_{2}, \ldots, A_{n}\right)=A$.

(2) Boundedness: assume $A^{-}=<\min _{j} T_{A_{j}}, \max _{j} I_{A_{j}}$, $\max _{j} F_{A_{j}}>$ and $A^{+}=<\max _{j} T_{A_{j}}, \min _{j} I_{A_{j}}$, $\min _{j} F_{A_{j}}>$ for all $j \in\{1,2, \ldots, n\}$, and then $A^{-} \subseteq \operatorname{SNNOWA}_{\omega}\left(A_{1}, A_{2}, \ldots, A_{n}\right) \subseteq A^{+}$.

(3) Monotonity: assume $A_{j}^{*}=<T_{A_{i}^{*}}, I_{A_{j}^{*}}, F_{A_{j}^{*}}>$ and $A_{j} \subseteq A_{j}^{*} \quad$ for any $j \in\{1,2, \ldots, n\}$, and then $\operatorname{SNNOWA}_{\omega}\left(A_{1}, A_{2}, \ldots, A_{n}\right) \subseteq$ $\operatorname{SNNOWA}_{\omega}\left(A_{1}^{*}, A_{2}^{*}, \ldots, A_{n}^{*}\right)$.

Proof: The proof is similar to that for Property 1.

Definition 15: Let $A_{j}=<T_{A_{j}}, I_{A_{j}}, F_{A_{j}}>\quad(j=$ $1,2, \ldots, n)$ be a collection of SNNs, and SNNOWG :

$$
\mathrm{SNN}^{n} \rightarrow \mathrm{SNN}
$$

$$
\operatorname{SNNOWG}_{\omega}\left(A_{1}, A_{2}, \ldots, A_{n}\right)=\prod_{j=1}^{n} A_{\sigma(j)}^{\omega_{j}},
$$

and thus the SNNOWG operator is called an simplified neutrosophic number ordered weighted geometric operator of dimension $n$, where $A_{\sigma(j)}$ is the $j$ th largest value and $\omega=\left(\omega_{1}, \omega_{2}, \ldots, \omega_{n}\right)$ is the aggregation-associated vector such that . $(j=1,2, \ldots, n)$ and $\sum_{j=1}^{n} \omega_{j}=1$.

Theorem 5: Let $A_{j}=<T_{A_{j}}, I_{A_{j}}, F_{A_{j}}>(j=1,2, \ldots, n)$ be a collection of $S N N s$, and $\omega=\left(\omega_{1}, \omega_{2}, \ldots, \omega_{n}\right)$ be the aggregation-associated vector such that $\omega_{j} \geq 0(j=$ $1,2, \ldots, n)$ and $\sum_{j=1}^{n} \omega_{j}=1$. Then their aggregated result using the $S N N O W G$ operator is also an $S N N$, and

$$
\begin{aligned}
\operatorname{SNNOWG}_{\omega}\left(A_{1}, A_{2}, \ldots, A_{n}\right) \\
=\left\langle k^{-1}\left(\sum_{j=1}^{n} \omega_{j} k\left(T_{A_{\sigma(j)}}\right)\right),\right. \\
\left.\quad l^{-1}\left(\sum_{j=1}^{n} \omega_{j} l\left(I_{A_{\sigma(j)}}\right)\right), l^{-1}\left(\sum_{j=1}^{n} \omega_{j} l\left(F_{A_{\sigma(j)}}\right)\right)\right\rangle .
\end{aligned}
$$

Assume $k(x)=-\log (x)$, and then Theorem 5 can be represented by

$$
\begin{aligned}
& \operatorname{SNNOWG}_{\omega}\left(A_{1}, A_{2}, \ldots, A_{n}\right)=\left\langle\prod_{j=1}^{n} T_{A_{\sigma(j)}}^{\omega_{j}},\right. \\
& \left.1-\prod_{j=1}^{n}\left(1-I_{A_{\sigma(j)}}\right)^{\omega_{j}}, 1-\prod_{j=1}^{n}\left(1-F_{A_{\sigma(j)}}\right)^{\omega_{j}}\right\rangle .
\end{aligned}
$$

Let $k(x)=\log \left(\frac{2-x}{x}\right)$, and then Theorem 5 can be denoted by

$$
\begin{aligned}
& \text { SNNOWG }_{\omega}\left(A_{1}, A_{2}, \ldots, A_{n}\right) \\
& =\left\langle\frac{2 \prod_{j=1}^{n} T_{A_{\sigma(j)}}^{\omega_{j}}}{\prod_{j=1}^{n}\left(2-T_{A_{\sigma(j)}}\right)^{\omega_{j}}+\prod_{j=1}^{n} T_{A_{\sigma_{(j)}}}^{\omega_{j}}},\right. \\
& \quad \times \frac{\prod_{j=1}^{n}\left(1+I_{\left.A_{\sigma(j)}\right)}\right)^{\omega_{j}}-\prod_{j=1}^{n}\left(1-I_{A_{\sigma(j)}}\right)^{\omega_{j}}}{\prod_{j=1}^{n}\left(1+I_{\left.A_{\sigma(j)}\right)}\right)^{\omega_{j}}+\prod_{j=1}^{n}\left(1-I_{A_{\sigma(j)}}\right)^{\omega_{j}}}, \\
& \\
& \left.\quad \frac{\prod_{j=1}^{n}\left(1+F_{A_{\sigma(j)}}\right)^{\omega_{j}}-\prod_{j=1}^{n}\left(1-F_{A_{\sigma(j)}}\right)^{\omega_{j}}}{\prod_{j=1}^{n}\left(1+F_{A_{\sigma(j)}}\right)^{\omega_{j}}+\prod_{j=1}^{n}\left(1-F_{A_{\sigma(j)}}\right)^{\omega_{j}}}\right\rangle,
\end{aligned}
$$

where $A_{\sigma(j)}$ is the $j$ th largest value according to the total order $A_{\sigma(1)} \geq A_{\sigma(2)} \geq \cdots \geq A_{\sigma(n)}$.

Proof: Theorem 5 can be proved by the mathematical induction method, and the process is omitted here. 
Property 4: Let $A_{j}=<T_{A_{j}}, I_{A_{j}}, F_{A_{j}}>(j=1,2, \ldots, n)$ be a collection of SNNs.

(1) Idempotency: if all $A_{j}(j=1,2, \ldots, n)$ are equal, i.e., $A_{j}=A$, for all $j \in\{1,2, \ldots, n\}$, then $\mathrm{SNNOWG}_{\omega}\left(A_{1}, A_{2}, \ldots, A_{n}\right)=A$.

(2) Boundedness: assume $A^{-}=<\min _{j} T_{A_{j}}, \max _{j} I_{A_{j}}$, $\max _{j} F_{A_{j}}>\quad$ and $A^{+}=<\max _{j} T_{A_{j}}, \min _{j} I_{A_{j}}$, $\min _{j} F_{A_{j}}>$ for all $j \in\{1,2, \ldots, n\}$, and then $A^{-} \subseteq \operatorname{SNNOWG}_{\omega}\left(A_{1}, A_{2}, \ldots, A_{n}\right) \subseteq A^{+}$.

(3) Monotonity: assume $A_{j}^{*}=<T_{A_{j}^{*}}, I_{A_{j}^{*}}, F_{A_{j}^{*}}>$ and $A_{j} \subseteq A_{j}^{*} \quad$ for any $j \in\{1,2, \ldots, n\}$, and then $\operatorname{SNNOWG}_{\omega}\left(A_{1}, A_{2}, \ldots, A_{n}\right)$ $\subseteq \operatorname{SNNOWG}_{\omega}\left(A_{1}^{*}, A_{2}^{*}, \ldots, A_{n}^{*}\right)$.

Proof: The proof is similar to that for Property 1.

Definition 16: Let $A_{j}=<T_{A_{j}}, I_{A_{j}}, F_{A_{j}}>\quad(j=$ $1,2, \ldots, n)$ be a collection of SNNs, SNNHOWA : $\mathrm{SNN}^{n} \rightarrow \mathrm{SNN}$,

$$
\text { SNNHOW } A_{\omega}\left(A_{1}, A_{2}, \ldots, A_{n}\right)=\sum_{j=1}^{n} \omega_{j} \dot{A}_{\sigma(j)} \text {, }
$$

and then the SNNHOWA operator is called the simplified neutrosophic number hybrid ordered weighted averaging operator of dimension $n$, where $\dot{A}_{\sigma(j)}$ is the $j$ th largest of the weighted value $\dot{A}_{j}\left(\dot{A}_{j}=n w_{j} A_{j}, j=\right.$ $1,2, \ldots, n), w=\left(w_{1}, w_{2}, \ldots, w_{n}\right)$ is the weight vector of $A_{j}(j=1,2, \ldots, n)$, with $w_{j} \geq 0(j=1,2, \ldots, n)$ and $\sum_{j=1}^{n} w_{j}=1, \omega=\left(\omega_{1}, \omega_{2}, \ldots, \omega_{n}\right)$ is the aggregationassociated vector such that $\omega_{j} \geq 0(j=1,2, \ldots, n)$ and $\sum_{j=1}^{n} \omega_{j}=1$, and $n$ is the balancing coefficient.

Theorem 6: Let $A_{j}=<T_{A_{j}}, I_{A_{j}}, F_{A_{j}}>(j=1,2, \ldots, n)$ be a collection of SNNs, $w=\left(w_{1}, w_{2}, \ldots, w_{n}\right)$ be the weight vector of $A_{j}(j=1,2, \ldots, n)$, with $w_{j} \geq 0(j=$ $1,2, \ldots, n)$ and $\sum_{j=1}^{n} w_{j}=1$, and $\omega=\left(\omega_{1}, \omega_{2}, \ldots, \omega_{n}\right)$ be the aggregation-associated vector such that $\omega_{j} \geq 0$ $(j=1,2, \ldots, n)$ and $\sum_{j=1}^{n} \omega_{j}=1$. Then their aggregated result using the SNNHOWA operator is also an SNN, and

$$
\begin{gathered}
\operatorname{SNNHOWA}_{w}\left(A_{1}, A_{2}, \ldots, A_{n}\right)=\left\langle l^{-1}\left(\sum_{j=1}^{n} \omega_{j} l\left(T_{\dot{A}_{\sigma(j)}}\right)\right),\right. \\
\left.k^{-1}\left(\sum_{j=1}^{n} \omega_{j} k\left(I_{\dot{A}_{\sigma(j)}}\right)\right), k^{-1}\left(\sum_{j=1}^{n} \omega_{j} k\left(F_{\dot{A}_{\sigma(j)}}\right)\right)\right\rangle .
\end{gathered}
$$

Let $k(x)=-\log (x)$, and then Theorem 6 can be represented by

$$
\operatorname{SNNHOWA}_{\omega}\left(A_{1}, A_{2}, \ldots, A_{n}\right)
$$

$$
=\left\langle 1-\prod_{j=1}^{n}\left(1-T_{\dot{A}_{\sigma(j)}}\right)^{\omega_{j}}, \prod_{j=1}^{n} I_{\dot{A}_{\sigma(j)}}^{\omega_{j}}, \prod_{j=1}^{n} F_{\dot{A}_{\sigma(j)}}^{\omega_{j}}\right\rangle .
$$
by

If $k(x)=\log \left(\frac{2-x}{x}\right)$, then Theorem 6 can be represented

$$
\begin{aligned}
& \text { SNNHOWA }_{\omega}\left(A_{1}, A_{2}, \ldots, A_{n}\right) \\
& =\left\langle\frac{\prod_{j=1}^{n}\left(1+T_{\dot{A}_{\sigma(j)}}\right)^{\omega_{j}}-\prod_{j=1}^{n}\left(1-T_{\dot{A}_{\sigma(j)}}\right)^{\omega_{j}}}{\prod_{j=1}^{n}\left(1+T_{\dot{A}_{\sigma(j)}}\right)^{\omega_{j}}+\prod_{j=1}^{n}\left(1-T_{\dot{A}_{\sigma(j)}}\right)^{\omega_{j}}},\right. \\
& \quad \times \frac{2 \prod_{j=1}^{n} I_{\dot{A}_{\sigma(j)}}^{\omega_{j}}}{\prod_{j=1}^{n}\left(2-I_{\dot{A}_{\sigma(j)}}\right)^{\omega_{j}}+\prod_{j=1}^{n} I_{\dot{A}_{\sigma(j)}}^{\omega_{j}}}, \\
& \left.\quad \times \frac{2 \prod_{j=1}^{n} F_{\dot{A}_{\sigma(j)}}^{\omega_{j}}}{\prod_{j=1}^{n}\left(2-F_{\dot{A}_{\sigma(j)}}\right)^{\omega_{j}}+\prod_{j=1}^{n} F_{\dot{A}_{\sigma(j)}}^{\omega_{j}}}\right\rangle
\end{aligned}
$$

where $\dot{A}_{\sigma(j)}$ is the $j$ th largest of the weighted value $\dot{A}_{j}\left(\dot{A}_{j}=\right.$ $\left.n w_{j} A_{j}, j=1,2, \ldots, n\right)$.

Proof: Theorem 6 can be proved by the mathematical induction method, and the process is omitted here.

Property 5: Let $A_{j}=<T_{A_{j}}, I_{A_{j}}, F_{A_{j}}>(j=$ $1,2, \ldots, n)$ be a collection of SNNs.

(1) Idempotency: if all $A_{j}(j=1,2, \ldots, n)$ are equal, i.e., $A_{j}=A$, for all $j \in\{1,2, \ldots, n\}$, then $\operatorname{SNNHOWA}_{\omega}\left(A_{1}, A_{2}, \ldots, A_{n}\right)=A$.

(2) Boundedness: assume $A^{-}=<\min _{j} T_{A_{j}}, \max _{j} I_{A_{j}}$, $\max _{j} F_{A_{j}}>$ and $A^{+}=<\max _{j} T_{A_{j}}, \min _{j} I_{A_{j}}$, $\min _{j} F_{A_{j}}>$ for all $j \in\{1,2, \ldots, n\}$, and then $A^{-} \subseteq \operatorname{SNNHOWA}_{\omega}\left(A_{1}, A_{2}, \ldots, A_{n}\right) \subseteq A^{+}$.

(3) Monotonity: assume $A_{j}^{*}=<T_{A_{j}^{*}}, I_{A_{j}^{*}}, F_{A_{j}^{*}}>$ and $A_{j} \subseteq A_{j}^{*} \quad$ for any $j \in\{1,2, \ldots, n\}$, and then $\operatorname{SNNHOWA}_{\omega}\left(A_{1}, A_{2}, \ldots, A_{n}\right)$ $\subseteq \operatorname{SNNHOWA}_{\omega}\left(A_{1}^{*}, A_{2}^{*}, \ldots, A_{n}^{*}\right)$.

Proof: The proof is similar to that for Property 1 .

Definition 17: Let $A_{j}=<T_{A_{j}}, I_{A_{j}}, F_{A_{j}}>\quad(j=$ $1,2, \ldots, n)$ be a collection of SNNs, and SNNHOWG : $\mathrm{SNN}^{n} \rightarrow \mathrm{SNN}$

$$
\operatorname{SNNHOWG}_{w}\left(A_{1}, A_{2}, \ldots, A_{n}\right)=\prod_{j=1}^{n} \dot{A}_{\sigma(j)}^{w_{j}},
$$

and then the SNNHOWG operator is called the simplified neutrosophic number hybrid ordered weighted geometric operator of dimension $n$, where $\dot{A}_{\sigma(j)}$ is the $j$ th largest of the weighted value $\dot{A}_{j}\left(\dot{A}_{j}=n w_{j} A_{j}, j=\right.$ $1,2, \ldots, n), w=\left(w_{1}, w_{2}, \ldots, w_{n}\right)$ is the weight vector of $A_{j}(j=1,2, \ldots, n)$, with $w_{j} \geq 0(j=1,2, \ldots, n)$ and $\sum_{j=1}^{n} w_{j}=1, \omega=\left(\omega_{1}, \omega_{2}, \ldots, \omega_{n}\right)$ is the aggregationassociated vector such that $\omega_{j} \geq 0(j=1,2, \ldots, n)$ and $\sum_{j=1}^{n} \omega_{j}=1$, and $n$ is the balancing coefficient. 
Theorem 7: Let $A_{j}=<T_{A_{j}}, I_{A_{j}}, F_{A_{j}}>\quad(j=$ $1,2, \ldots, n)$ be a collection of SNNs, $w=\left(w_{1}, w_{2}, \ldots, w_{n}\right)$ be the vector of $A_{j}(j=1,2, \ldots, n)$, with $w_{j} \in[0,1]$ and $\sum_{j=1}^{n} w_{j}=1$, and $\omega=\left(\omega_{1}, \omega_{2}, \ldots, \omega_{n}\right)$ be the aggregation-associated vector such that $\omega_{j} \geq 0$ $(j=1,2, \ldots, n)$ and $\sum_{j=1}^{n} \omega_{j}=1$. Then their aggregated result using the $S N N H O W G$ operator is also an $S N N$, and

$$
\begin{aligned}
& \operatorname{SNNHOWG}_{\omega}\left(A_{1}, A_{2}, \ldots, A_{n}\right) \\
& \quad=\left\langle k^{-1}\left(\sum_{j=1}^{n} \omega_{j} k\left(T_{\dot{A}_{\sigma(j)}}\right)\right),\right. \\
& \left.l^{-1}\left(\sum_{j=1}^{n} \omega_{j} l\left(I_{\dot{A}_{\sigma(j)}}\right)\right), l^{-1}\left(\sum_{j=1}^{n} \omega_{j} l\left(F_{\dot{A}_{\sigma(j)}}\right)\right)\right\rangle .
\end{aligned}
$$

Assume $k(x)=-\log (x)$, and then Theorem 7 can be represented by

$$
\begin{gathered}
\operatorname{SNNHOWG}_{\omega}\left(A_{1}, A_{2}, \ldots, A_{n}\right)=\left\langle\prod_{j=1}^{n} T_{\dot{A}_{\sigma(j)}}^{\omega_{j}},\right. \\
\left.1-\prod_{j=1}^{n}\left(1-I_{\dot{A}_{\sigma(j)}}\right)^{\omega_{j}}, 1-\prod_{j=1}^{n}\left(1-F_{\dot{A}_{\sigma(j)}}\right)^{\omega_{j}}\right\rangle .
\end{gathered}
$$

Let $k(x)=\log \left(\frac{2-x}{x}\right)$, and then Theorem 7 can be denoted by

$$
\begin{aligned}
& \mathrm{SNNHOWG}_{\omega}\left(A_{1}, A_{2}, \ldots, A_{n}\right) \\
& =\left\langle\frac{2 \prod_{j=1}^{n} T_{\dot{A}_{\sigma(j)}}^{\omega_{j}}}{\prod_{j=1}^{n}\left(2-T_{\dot{A}_{\sigma(j)}}\right)^{\omega_{j}}+\prod_{j=1}^{n} T_{\dot{A}_{\sigma(j)}}^{\omega_{j}}},\right. \\
& \times \frac{\prod_{j=1}^{n}\left(1+I_{\dot{A}_{\sigma(j)}}\right)^{\omega_{j}}-\prod_{j=1}^{n}\left(1-I_{\dot{A}_{\sigma(j)}}\right)^{\omega_{j}}}{\prod_{j=1}^{n}\left(1+I_{\dot{A}_{\sigma(j)}}\right)^{\omega_{j}}+\prod_{j=1}^{n}\left(1-I_{\dot{A}_{\sigma(j)}}\right)^{\omega_{j}}}, \\
& \left.\times \frac{\prod_{j=1}^{n}\left(1+F_{\dot{A}_{\sigma(j)}}\right)^{\omega_{j}}-\prod_{j=1}^{n}\left(1-F_{\dot{A}_{\sigma(j)}}\right)^{\omega_{j}}}{\prod_{j=1}^{n}\left(1+F_{\dot{A}_{\sigma(j)}}\right)^{\omega_{j}}+\prod_{j=1}^{n}\left(1-F_{\dot{A}_{\sigma(j)}}\right)^{\omega_{j}}}\right\rangle,
\end{aligned}
$$

where $A_{\sigma(j)}$ is the $j$ th largest value according to the total or$\operatorname{der} A_{\sigma(1)} \geq A_{\sigma(2)} \geq \cdots \geq A_{\sigma(n)}$ and $\dot{A}_{\sigma(j)}$ is the $j$ th largest of the weighted value $\dot{A}_{j}\left(\dot{A}_{j}=A j^{n w j}, j=1,2, \ldots, n\right)$.

Proof: Theorem 7 can be proved by the mathematical induction method and the process is omitted here.

Property 6: Let $A_{j}=<T_{A_{j}}, I_{A_{j}}, F_{A_{j}}>(j=$ $1,2, \ldots, n)$ be a collection of SNNs.

(1) Idempotency: if all $A_{j}(j=1,2, \ldots, n)$ are equal, i.e., $A_{j}=A$, for all $j \in\{1,2, \ldots, n\}$, then $\mathrm{SNNHOWG}_{\omega}\left(A_{1}, A_{2}, \ldots, A_{n}\right)=A$.
(2) Boundedness: assume $A^{-}=<\min _{j} T_{A_{j}}, \max _{j} I_{A_{j}}$, $\max _{j} F_{A_{j}}>\quad$ and $A^{+}=<\max _{j} T_{A_{j}}, \min _{j} I_{A_{j}}$, $\min _{j} F_{A_{j}}>$ for all $j \in\{1,2, \ldots, n\}$, and then $A^{-} \subseteq \operatorname{SNNHOWG}_{\omega}\left(A_{1}, A_{2}, \ldots, A_{n}\right) \subseteq A^{+}$.

(3) Monotonity: assume $A_{j}^{*}=<T_{A_{j}^{*}}, I_{A_{j}^{*}}, F_{A_{j}^{*}}>$ and $A_{j} \subseteq A_{j}^{*} \quad$ for any $j \in\{1,2, \ldots, n\}$, and then $\operatorname{SNNHOWG}_{\omega}\left(A_{1}, A_{2}, \ldots, A_{n}\right)$ $\subseteq \mathrm{SNNHOWG}_{\omega}\left(A_{1}^{*}, A_{2}^{*}, \ldots, A_{n}^{*}\right)$.

Proof: The proof is similar to that for Property 1.

Definition 18: Let $A_{j}=<T_{A_{j}}, I_{A_{j}}, F_{A_{j}}>\quad(j=$ $1,2, \ldots, n)$ be a collection of SNNs, and GSNNWA: $\mathrm{SNN}^{\mathrm{n}} \rightarrow \mathrm{SNN}$,

$$
\operatorname{GSNNWA}_{w}\left(A_{1}, A_{2}, \ldots, A_{n}\right)=\left(\sum_{j=1}^{n} w_{j}\left(A_{j}\right)^{\lambda}\right)^{1 / \lambda}
$$

and then the GSNNWA operator is called the generalised simplified neutrosophic number weighted averaging operator of dimension $n$, where $w=\left(w_{1}, w_{2}, \ldots, w_{n}\right)$ is the weight vector of $A_{j}(j=1,2, \ldots, n)$ with $w_{j}>0(j=$ $1,2, \ldots, n)$ and $\sum_{j=1}^{n} w_{j}=1$.

Theorem 8: Let $\quad A_{j}=<T_{A_{j}}, I_{A_{j}}, F_{A_{j}}>$ $(j=1,2, \ldots, n)$ be a collection of SNNs, and $w=\left(w_{1}, w_{2}, \ldots, w_{n}\right)$ be the weight vector of $A_{j}$ $(j=1,2, \ldots, n)$, with $\lambda>0, w_{j} \geq 0(j=1,2, \ldots, n)$ and $\sum_{j=1}^{n} w_{j}=1$. Then their aggregated result using the $S N N H O W G$ operator is also an $S N N$, and

$$
\begin{aligned}
\operatorname{GSNNWA}_{w}\left(A_{1}, A_{2}, \ldots, A_{n}\right) \\
=\left\langlek ^ { - 1 } \left(\frac{1}{\lambda} k\left(l^{-1}\left(\sum_{j=1}^{n} w_{j} l\left(k^{-1}\left(\lambda k\left(T_{j}\right)\right)\right)\right)\right),\right.\right. \\
l^{-1}\left(\frac{1}{\lambda} l\left(k^{-1}\left(\sum_{j=1}^{n} w_{j} k\left(l^{-1}\left(\lambda l\left(I_{j}\right)\right)\right)\right)\right),\right. \\
l^{-1}\left(\frac{1}{\lambda} l\left(k^{-1}\left(\sum_{j=1}^{n} w_{j} k\left(l^{-1}\left(\lambda l\left(F_{j}\right)\right)\right)\right)\right)\right) .
\end{aligned}
$$

Assume $k(x)=-\log (x)$, and then Theorem 8 can be represented by

$$
\begin{aligned}
& \operatorname{GSNNWA}_{w}\left(A_{1}, A_{2}, \ldots, A_{n}\right) \\
& =\left\langle\left(1-\prod_{j=1}^{n}\left(1-\left(T_{j}\right)^{\lambda}\right)^{w_{j}}\right)^{1 / \lambda},\right.
\end{aligned}
$$




$$
\begin{aligned}
& 1-\left(1-\prod_{j=1}^{n}\left(1-\left(1-I_{j}\right)^{\lambda}\right)^{w_{j}}\right)^{1 / \lambda} \\
& \left.1-\left(1-\prod_{j=1}^{n}\left(1-\left(1-F_{j}\right)^{\lambda}\right)^{w_{j}}\right)^{1 / \lambda}\right\rangle .
\end{aligned}
$$

Let $k(x)=\log \left(\frac{2-x}{x}\right)$, and then Theorem 8 can be denoted by

$$
\begin{aligned}
& \operatorname{GSNNWA}_{w}\left(A_{1}, A_{2}, \ldots, A_{n}\right) \\
& =\left[\frac{2}{\left(\frac{\prod_{j=1}^{n}\left(\alpha_{A_{j}}\right)^{w_{j}}+3}{\prod_{j=1}^{n}\left(\alpha_{A_{j}}\right)^{w_{j}}-1}\right)^{1 / \lambda}+1}, 1-\frac{2}{\left(\frac{\prod_{j=1}^{n}\left(\beta_{A_{j}}\right)^{w_{j}}+3}{\prod_{j=1}^{n}\left(\beta_{A_{j}}\right)^{\omega_{j}}-1}\right)^{1 / \lambda}+1},\right. \\
& \left.1-\frac{2}{\left(\frac{\prod_{j=1}^{n}\left(\gamma_{A_{j}}\right)^{w_{j}}+3}{\prod_{j=1}^{n}\left(\gamma_{A_{j}}\right)^{w_{j}}-1}\right)^{1 / \lambda}+1}\right],
\end{aligned}
$$

where

$\alpha_{A_{j}}=\left(\frac{1+\frac{2}{\left(\frac{2-T_{A_{j}}}{T_{A_{j}}}\right)^{\lambda}+1}}{1-\frac{2}{\left(\frac{2-T_{A_{j}}}{T_{A_{j}}}\right)^{\lambda}+1}}\right), \beta_{A_{j}}=\left(\frac{1+\frac{2}{\left(\frac{1+I_{A_{j}}}{1-I_{A_{j}}}\right)^{\lambda}+1}}{1-\frac{2}{\left(\frac{1+I_{A_{j}}}{1-I_{A_{j}}}\right)^{\lambda}+1}}\right)$,

$\gamma_{A_{j}}=\left(\frac{1+\frac{2}{\left(\frac{1+F_{A_{j}}}{1-F_{A_{j}}}\right)^{\lambda}+1}}{1-\frac{2}{\left(\frac{1+F_{A_{j}}}{1-F_{A_{j}}}\right)^{\lambda}+1}}\right)$.

Apparently, if $\lambda=1$, then the GSNNWA operator is reduced to the SNNWA operator.

Definition 19: Let $A_{j}=<T_{A_{j}}, I_{A_{j}}, F_{A_{j}}>\quad(j=$ $1,2, \ldots, n)$ be a collection of SNNs, and GSNNWG: $\mathrm{SNN}^{n} \rightarrow \mathrm{SNN}$,

$$
\operatorname{GSNNWG}_{w}\left(A_{1}, A_{2}, \ldots, A_{n}\right)=\frac{1}{\lambda}\left(\prod_{j=1}^{n}\left(\lambda A_{j}\right)^{w_{j}}\right),
$$

and then the GSNNWG operator is called the generalised simplified neutrosophic number weighted geometric operator of dimension $n$, where $w=\left(w_{1}, w_{2}, \ldots, w_{n}\right)$ is the weight vector of $A_{j}(j=1,2, \ldots, n)$ with $w_{j}>0(j=$ $1,2, \ldots, n)$ and $\sum_{j=1}^{n} w_{j}=1$.

Theorem 9: Let $A_{j}=<T_{A_{j}}, I_{A_{j}}, F_{A_{j}}>(j=1,2, \ldots, n)$ be a collection of $S N N s$, and $w=\left(w_{1}, w_{2}, \ldots, w_{n}\right)$ be the weight vector of $A_{j}(j=1,2, \ldots, n)$, with $\lambda>0, w_{j} \geq 0$ $(j=1,2, \ldots, n)$ and $\sum_{j=1}^{n} w_{j}=1$. Then their aggregated result using the SNNHOWG operator is also an $S N N$, and

$$
\begin{aligned}
\operatorname{GSNNWG}_{w}\left(A_{1}, A_{2}, \ldots, A_{n}\right) \\
=\left\langlel ^ { - 1 } \left(\frac{1}{\lambda} l\left(k^{-1}\left(\sum_{j=1}^{n} w_{j} k\left(l^{-1}\left(\lambda l\left(T_{A_{j}}\right)\right)\right)\right)\right),\right.\right. \\
\times k^{-1}\left(\frac{1}{\lambda} k\left(l^{-1}\left(\sum_{j=1}^{n} w_{j} l\left(k^{-1}\left(\lambda k\left(I_{A_{j}}\right)\right)\right)\right)\right),\right. \\
k^{-1}\left(\frac{1}{\lambda} k\left(l^{-1}\left(\sum_{j=1}^{n} w_{j} l\left(k^{-1}\left(\lambda k\left(F_{A_{j}}\right)\right)\right)\right)\right)\right\rangle .
\end{aligned}
$$

Assume $k(x)=-\log (x)$, and then Theorem 9 can be represented by

$$
\begin{aligned}
& \operatorname{GSNNWG}_{w}\left(A_{1}, A_{2}, \ldots, A_{n}\right) \\
& =\left\langle 1-\left(1-\prod_{j=1}^{n}\left(1-\left(1-T_{A_{j}}\right)^{\lambda}\right)^{w_{j}}\right)^{1 / \lambda},\right. \\
& \left.\left.\times\left(1-\prod_{j=1}^{n}\left(1-\left(I_{A_{j}}\right)^{\lambda}\right)^{w_{j}}\right)^{1 / \lambda},\left(1-\prod_{j=1}^{n}\left(1-\left(F_{A_{j}}\right)^{\lambda}\right)^{w_{j}}\right)^{1 / \lambda}\right]\right\rangle .
\end{aligned}
$$

Let $k(x)=\log \left(\frac{2-x}{x}\right)$, and then Theorem 8 can be denoted by

$$
\begin{aligned}
& \operatorname{GSNNWG}_{w}\left(A_{1}, A_{2}, \ldots, A_{n}\right) \\
& =\left[1-\frac{2}{\left(\frac{\prod_{j=1}^{n}\left(\gamma_{A_{j}} w_{j}+3\right.}{\prod_{j=1}^{n}\left(\gamma_{A_{j}}\right)^{w_{j}}-1}\right)^{1 / \lambda}+1}, \frac{2}{\left(\frac{\prod_{j=1}^{n}\left(\alpha_{A_{j}}\right)^{w_{j}}+3}{\prod_{j=1}^{n}\left(\alpha_{A_{j}}\right)^{w_{j}}-1}\right)^{1 / \lambda}+1},\right. \\
& \left.\times \frac{2}{\left(\frac{\prod_{j=1}^{n}\left(\beta_{A_{j}}\right)^{w_{j}}+3}{\prod_{j=1}^{j}\left(\beta_{A_{j}}\right)^{w_{j}}-1}\right)^{1 / \lambda}+1}\right],
\end{aligned}
$$

where

$$
\begin{aligned}
& \gamma_{A_{j}}=\left(\frac{1+\frac{2}{\left(\frac{1+T_{A_{j}}}{1-T_{A_{j}}}\right)^{\lambda}+1}}{1-\frac{2}{\left(\frac{1+T_{A_{j}}}{1-T_{A_{j}}}\right)^{\lambda}+1}}\right), \alpha_{A_{j}}=\left(\frac{1+\frac{2}{\left(\frac{2-I_{A_{j}}}{I_{A_{j}}}\right)^{\lambda}+1}}{1-\frac{2}{\left(\frac{2-I_{A_{j}}}{I_{A_{j}}}\right)^{\lambda}+1}}\right) \text {, } \\
& \beta_{A_{j}}=\left(\frac{1+\frac{2}{\left(\frac{2-F_{A_{j}}}{F_{A_{j}}}\right)^{\lambda}+1}}{1-\frac{2}{\left(\frac{2-F_{A_{j}}}{F_{A_{j}}}\right)^{\lambda}+1}}\right) \text {. }
\end{aligned}
$$


Apparently, if $\lambda=1$, then the GSNNWG operator is reduced to the SNNWG operator.

Similarly, it can be proved that the GSNNWA and GSNNWG operators have the same properties as the SNNWA operator.

\subsection{The MCGDM method based on the aggregation operators of $S N N s$}

Assume that there are $n$ alternatives $A=\left\{a_{1}, a_{2}, \ldots, a_{n}\right\}$ and $m$ criteria $C=\left\{c_{1}, c_{2}, \ldots, c_{m}\right\}$, and the weight vector of criteria is $w=\left(w_{1}, w_{2}, \ldots, w_{m}\right)$, where $w_{j} \geq 0$ $(j=1,2, \ldots, m), \sum_{j=1}^{m} w_{j}=1$. Suppose that there are $k$ decision-makers $D=\left\{d_{1}, d_{2}, \ldots, d_{k}\right\}$, whose corresponding weight vector is $\lambda=\left(\lambda_{1}, \lambda_{2}, \ldots, \lambda_{k}\right)$. Let $R=\left(a_{i j}^{k}\right)_{n \times m}$ be the simplified neutrosophic decision matrix, where $a_{i j}^{k}=<T_{a_{i j}^{k}}, I_{a_{i j}^{k}}, F_{a_{i j}^{k}}>$ is the value of a criterion, denoted by SNNs, where $T_{a_{i j}^{k}}$ indicates the truth-membership function that the alternative $a_{i}$ satisfies the criterion $c_{j}$ for the $k$ th decision-maker, $I_{a_{i j}^{k}}$ indicates the indeterminacymembership function that the alternative $a_{i}$ satisfies the criterion $c_{j}$ for the $k$ th decision-maker, and $F_{a_{i j}^{k}}$ indicates the falsity-membership function that the alternative $a_{i}$ satisfies the criterion $c_{j}$ for the $k$ th decision-maker. This method uses the aggregation operators of SNNs in order to solve the MCGDM problem mentioned above.

In the following steps, a procedure for ranking and selecting the most desirable alternative(s) is provided.

\section{Step 1: Aggregate the SNNs of each decision-maker.}

Utilise the GSNNWA or GSNNWG operator to aggregate the SNNs of each decision-maker, and the individual value of the alternative $a_{i}(i=1,2, \ldots, n)$ can be obtained.

$$
\begin{aligned}
x_{i}= & \operatorname{GSNNWA}_{w}\left(a_{i 1}, a_{i 2}, \ldots, a_{i m}\right) \\
= & <l^{-1}\left(w_{1} l\left(T_{a_{i 1}}\right)+w_{2} l\left(T_{a_{i 2}}\right) \ldots+w_{n} l\left(T_{a_{i n}}\right)\right), \\
& k^{-1}\left(w_{1} k\left(I_{a_{i 1}}\right)+w_{2} k\left(I_{a_{i 2}}\right) \ldots+w_{n} k\left(I_{a_{i n}}\right)\right), \\
& k^{-1}\left(w_{1} k\left(F_{a_{i 1}}\right)+w_{2} k\left(F_{a_{i 2}}\right) \ldots+w_{n} k\left(F_{a_{i n}}\right)\right)>,
\end{aligned}
$$

or

$$
\begin{aligned}
x_{i}= & \operatorname{GSNNWG}_{w}\left(a_{i 1}, a_{i 2}, \ldots, a_{i m}\right) \\
= & <k^{-1}\left(w_{1} k\left(T_{a_{i 1}}\right)+w_{2} k\left(T_{a_{i 2}}\right) \ldots+w_{n} k\left(T_{a_{i m}}\right)\right), \\
& l^{-1}\left(w_{1} l\left(I_{a_{i 1}}\right)+w_{2} l\left(I_{a_{i 2}}\right) \ldots+w_{n} l\left(I_{a_{i m}}\right)\right), \\
& l^{-1}\left(w_{1} l\left(F_{a_{i 1}}\right)+w_{2} l\left(F_{a_{i 2}}\right) \ldots+w_{n} l\left(F_{a_{i m}}\right)\right)>.
\end{aligned}
$$

Step 2: Aggregate the SNNs of all decision-makers.

Utilise the SNNHOWA or SNNHOWG operator to obtain the overall SNN $y_{i}$ for all alternatives $a_{i}(i=$ $1,2, \ldots, n)$, i.e.,

$$
y_{i}=\operatorname{SNNHOWA}_{w}\left(x_{1}, x_{2}, \ldots, x_{k}\right)
$$

$$
\begin{aligned}
= & <l^{-1}\left(w_{1} l\left(T_{\dot{y}_{\sigma(1)}}\right)+w_{2} l\left(T_{\dot{y}_{\sigma(2)}}\right) \ldots+w_{n} l\left(T_{\dot{y}_{\sigma(k)}}\right)\right), \\
& k^{-1}\left(w_{1} k\left(I_{\dot{y}_{\sigma(1)}}\right)+w_{2} k\left(I_{\dot{y}_{\sigma(2)}}\right) \ldots+w_{n} k\left(I_{\dot{y}_{\sigma(k)}}\right)\right), \\
& k^{-1}\left(w_{1} k\left(F_{\dot{y}_{\sigma(1)}}\right)+w_{2} k\left(F_{\dot{y}_{\sigma(2)}}\right) \ldots+w_{n} k\left(F_{\dot{y}_{\sigma(k)}}\right)\right)>
\end{aligned}
$$

or

$$
\begin{aligned}
y_{i}= & \operatorname{SNNHOWG}_{w}\left(x_{1}, x_{2}, \ldots, x_{k}\right) \\
= & <k^{-1}\left(w_{1} k\left(T_{\dot{y}_{\sigma(1)}}\right)+w_{2} k\left(T_{\dot{y}_{\sigma(2)}}\right) \ldots+w_{n} k\left(T_{\dot{y}_{\sigma(k)}}\right)\right), \\
& l^{-1}\left(w_{1} l\left(I_{\dot{y}_{\sigma(1)}}\right)+w_{2} l\left(I_{\dot{y}_{\sigma(2)}}\right) \ldots+w_{n} l\left(I_{\dot{y}_{\sigma(k)}}\right)\right), \\
& l^{-1}\left(w_{1} l\left(F_{\dot{y}_{\sigma(1)}}\right)+w_{2} l\left(F_{\dot{y}_{\sigma(2)}}\right) \ldots+w_{n} l\left(F_{\dot{y}_{\sigma(k)}}\right)\right)>.
\end{aligned}
$$

Step 3: Calculate the score function value $s\left(y_{i}\right)$, accuracy function value $a\left(y_{i}\right)$ and certainty function value $c\left(y_{i}\right)$ of $y_{i}$ $(i=1,2, \ldots, m)$ using Definition 10.

Step 4: Rank the alternatives.

According to Definition 11, an order of priority for all the alternatives $a_{i}(i=1,2, \ldots, m)$ could be obtained and the best one can be chosen.

\section{An illustrative example}

In this section, an example of MCGDM problems is used to demonstrate the applicability and effectiveness of the proposed decision-making method.

Let us consider a decision-making problem adapted from Lupiáñez (2009). There is an investment company, which wants to invest a sum of money in the best option. There are four possible alternatives to invest the money: (1) $A_{1}$ is a car company; (2) $A_{2}$ is a food company; (3) $A_{3}$ is a computer company and (4) $A_{4}$ is an arms company. The investment company must make a decision according to the following three criteria: (1) $C_{1}$ is the risk analysis; (2) $C_{2}$ is the growth analysis and (3) $C_{3}$ is the environmental impact analysis, where $C_{1}$ and $C_{2}$ are of the maximising type, and $C_{3}$ is a minimising type. The weight vector of the criteria is given by $w=(0.35,0.25,0.4)$. Suppose that there are three decision-makers, $\left\{d_{1}, d_{2}, d_{3}\right\}$ whose corresponding weight vector is $\lambda=(0.5,0.3,0.2)$. The four possible alternatives are to be evaluated under these three criteria and are in the form of SNNs for each decision-maker, as shown in the following simplified neutrosophic decision matrix $D$ :

$D^{1}=\left(\begin{array}{ccc}\langle 0.4,0.2,0.3\rangle & \langle 0.4,0.2,0.3\rangle & \langle 0.2,0.2,0.5\rangle \\ \langle 0.6,0.1,0.2\rangle & \langle 0.6,0.1,0.2\rangle & \langle 0.5,0.2,0.2\rangle \\ \langle 0.3,0.2,0.3\rangle & \langle 0.5,0.2,0.3\rangle & \langle 0.5,0.3,0.2\rangle \\ \langle 0.7,0.0,0.1\rangle & \langle 0.6,0.1,0.2\rangle & \langle 0.4,0.3,0.2\rangle\end{array}\right) ;$

$D^{2}=\left(\begin{array}{ccc}\langle 0.5,0.2,0.2\rangle & \langle 0.6,0.2,0.3\rangle & \langle 0.3,0.2,0.4\rangle \\ \langle 0.6,0.1,0.2\rangle & \langle 0.7,0.2,0.3\rangle & \langle 0.5,0.2,0.3\rangle \\ \langle 0.4,0.1,0.3\rangle & \langle 0.5,0.3,0.3\rangle & \langle 0.6,0.2,0.2\rangle \\ \langle 0.7,0.3,0.1\rangle & \langle 0.6,0.3,0.2\rangle & \langle 0.5,0.1,0.2\rangle\end{array}\right) ;$ 


$$
D^{3}=\left(\begin{array}{ccc}
\langle 0.5,0.1,0.2\rangle & \langle 0.5,0.2,0.2\rangle & \langle 0.3,0.1,0.3\rangle \\
\langle 0.5,0.3,0.2\rangle & \langle 0.7,0.1,0.3\rangle & \langle 0.5,0.3,0.3\rangle \\
\langle 0.6,0.2,0.3\rangle & \langle 0.5,0.1,0.3\rangle & \langle 0.5,0.1,0.2\rangle \\
\langle 0.5,0.3,0.2\rangle & \langle 0.7,0.2,0.2\rangle & \langle 0.7,0.2,0.2\rangle
\end{array}\right)
$$

\subsection{The decision-making procedure based on SNNs}

Step 1: Utilise the GSNNWA or GSNNWG operator to obtain the SNNs for each decision-maker. Because the aggregation results based on the GSNNWA and GSNNWG operators are different, they are calculated separately. For convenience, the operations of SNNs are based on the Algebraic t-conorm and t-norm and $\lambda=1$.

By using the GSNNWA operator, the alternatives matrix $A_{\mathrm{WA}}$ can be obtained:

$A_{\mathrm{WA}}=\left(\begin{array}{ll}<0.327,0.325,0.368> & <0.459,0.325,0.292> \\ <0.563,0.285,0.200> & <0.593,0.339,0.260> \\ <0.438,0.382,0.255> & <0.513,0.375,0.255> \\ <0.575,0.347,0.157> & <0.605,0.260,0.157>\end{array}\right.$

With the GSNNWG operator, the alternatives matrix $A_{\mathrm{WG}}$ is shown as follows:

$A_{\mathrm{WG}}=\left(\begin{array}{ll}<0.303,0.200,0.388> & <0.427,0.242,0.310> \\ <0.588,0.289,0.200> & <0.579,0.309,0.206> \\ <0.418,0.337,0.262> & <0.497,0.389,0.262> \\ <0.538,0.206,0.166> & <0.589,0.388,0.166>\end{array}\right.$

Step 2: Aggregate the SNNs of all decision-makers.

Utilise the SNNHOWA or SNNHOWG operator to obtain the overall SNN $y_{i}$ for all alternatives $a_{i}$ ( $i=$ $1,2, \ldots, n)$, with the SNNHOWA operator, the overall SNN can be obtained as follows:

$$
H_{\mathrm{WA}}=\left(\begin{array}{l}
<0.590,0.279,0.188> \\
<0.859,0.274,0.137> \\
<0.728,0.302,0.153> \\
<0.905,0.280,0.100>
\end{array}\right) .
$$

With the SNNHOWG operator, the overall SNN can be obtained as follows:

$$
H_{\mathrm{WG}}=\left(\begin{array}{c}
<0535,0.193,0.202> \\
<0.842,0.277,0.140> \\
<0.694,0.314,0.157> \\
<0.854,0.292,0.104>
\end{array}\right) .
$$

Step 3: Calculate the score function value, accuracy function value and certainty function value.
For the alternatives matrix $H_{\mathrm{WA}}$, using Definition 10, the score function of $H_{\mathrm{WA}}$ can be obtained:

$$
s\left(H_{\mathrm{WA}}\right)=(0.708,0.816,0.757,0.842) .
$$

For the alternatives matrix $H_{\mathrm{WG}}$, using Definition 10, the score function of $H_{\mathrm{WG}}$ is shown as follows:

$$
s\left(H_{\mathrm{WG}}\right)=(0.713,0.809,0.741,0.819) .
$$

It is clear that the score values are different; therefore, there is no need to compute the accuracy function value and certainty function value.

Step 4: Obtain an order of priority for the alternatives and choose the best one.

$$
\left.\begin{array}{l}
<0.428,0.257,0.235> \\
<0.560,0.307,0.260> \\
<0.538,0.207,0.255> \\
<0.641,0.310,0.200>
\end{array}\right)
$$

According to Definition 11 and the results in Step 3, for $A_{\mathrm{WA}}$, the final ranking is $A_{4} \succ A_{2} \succ A_{3} \succ A_{1}$. Clearly, the best alternative is $A 4$.

$$
\left.\begin{array}{l}
<0.408,0.210,0.242> \\
<0.544,0.348,0.267> \\
<0.533,0.317,0.262> \\
<0.622,0.484,0.200>
\end{array}\right) .
$$

Similarly, for $A_{\mathrm{WG}}$, the final ranking is $A_{4} \succ A_{2} \succ$ $A_{3} \succ A_{1}$. And the best alternative is $A_{1}$.

If $k(x)=\log \left(\frac{2-x}{x}\right)$ for $A_{\mathrm{WA}}$, then the ranking of the four alternatives is still $A_{4} \succ A_{2} \succ A_{3} \succ A_{1}$, and the ranking is $A_{4} \succ A_{2} \succ A_{3} \succ A_{1}$ for $A_{\mathrm{WG}}$.

\subsection{The sensitivity analysis}

In the following discussion, the influence of $\lambda$ on the ranking of alternatives is investigated. The results can be found in Table 1.

From Table 1, it can be seen that the rankings of the alternatives are slightly different as $\lambda$ changes. However, for the GSNNWA and GSNNWG operators, the best alternative is $A_{4}$, while the worst alternative is $A_{1}$ or $A_{3}$. The authors believe that $\lambda$ can be considered as a reflection of the decision-makers' preferences. Based on Archimedean t-conorm and t-norm, Beliakov et al. (2011) introduced some operations for IFSs, proposed two general concepts for constructing other types of aggregation operators for IFSs, which extended the existing methods, and demon- 
Table 1. The results of the sensitivity analysis.

\begin{tabular}{llcccccc}
\hline & \multicolumn{3}{c}{ GSNNWA } & & & \multicolumn{2}{c}{ GSNNWG } \\
\cline { 2 - 3 }$\lambda$ & The final ranking & The best one & The worst one & & The final ranking & The best one & The worst one \\
\hline$\lambda=1$ & $A_{4} \succ A_{2} \succ A_{3} \succ A_{1}$ & $A_{4}$ & $A_{1}$ & & $A_{4} \succ A_{2} \succ A_{3} \succ A_{1}$ & $A_{4}$ & $A_{1}$ \\
$\lambda=2$ & $A_{4} \succ A_{2} \succ A_{3} \succ A_{1}$ & $A_{4}$ & $A_{1}$ & & $A_{4} \succ A_{2} \succ A_{3} \succ A_{1}$ & $A_{4}$ & $A_{1}$ \\
$\lambda=5$ & $A_{4} \succ A_{2} \succ A_{1} \succ A_{3}$ & $A_{4}$ & $A_{3}$ & & $A_{4} \succ A_{2} \succ A_{1} \succ A_{3}$ & $A_{4}$ & $A_{3}$ \\
$\lambda=10$ & $A_{4} \succ A_{2} \succ A_{3} \succ A_{1}$ & $A_{4}$ & $A_{1}$ & & $A_{4} \succ A_{2} \succ A_{3} \succ A_{1}$ & $A_{4}$ & $A_{1}$ \\
$\lambda=20$ & $A_{4} \succ A_{2} \succ A_{3} \succ A_{1}$ & $A_{4}$ & $A_{1}$ & & $A_{4} \succ A_{2} \succ A_{3} \succ A_{1}$ & $A_{4}$ & $A_{1}$ \\
\hline
\end{tabular}

strated that the operators obtained by using Lukasiewicz t-norm are consistent with the ones for ordinary FSs. In order to calculate the actual aggregation values of the alternatives, different aggregation operators can be used. It is also found that those aggregation operators are all based on different $\mathrm{t}$-conorms and t-norms and are used to deal with different relationships of the aggregated arguments, which can provide more choices for decision-makers.

\subsection{The comparison analysis and discussion}

In order to validate the feasibility of the proposed decisionmaking method based on the aggregation operators of SNNs, a comparison study is now conducted.

(1) The similarity measure proposed by Majumdar and Samant (2014) is going to be utilised in this discussion. By using the proposed aggregation operators, the aggregated values that were presented in Step 1 of Subsection 5.1. can be obtained. According to the similarity measure (Majumdar \& Samant, 2014), the ideal alternative is $A^{*}=\langle 1,0,0\rangle$, thus the following results could be obtained.

With the GSNNWA operator $(\lambda=1)$,

$$
\begin{aligned}
& S_{1}\left(A_{1}, A^{*}\right)=0.402 ; S_{2}\left(A_{2}, A^{*}\right)=0.609 ; \\
& S_{3}\left(A_{3}, A^{*}\right)=0.500 \text { and } S_{4}\left(A_{4}, A^{*}\right)=0.656 .
\end{aligned}
$$

With the GSNNWG operator $(\lambda=1)$,

$$
\begin{aligned}
& S_{1}\left(A_{1}, A^{*}\right)=0.384 ; S_{2}\left(A_{2}, A^{*}\right)=0.594 ; \\
& S_{3}\left(A_{3}, A^{*}\right)=0.472 \text { and } S_{4}\left(A_{4}, A^{*}\right)=0.612 .
\end{aligned}
$$

Therefore, the final ranking is still $A_{4} \succ A_{2} \succ A_{3} \succ$ $A_{1}$, and the best one is $A_{4}$.

(1) Similarly, if the operations and correlation coefficient are used (Ye, 2013a), then the final ranking is $A_{2} \succ A_{4} \succ A_{3} \succ A_{1}$, and the best one is $A_{2}$.
(2) If the operations and cross-entropy are utilised (Ye, $2014 \mathrm{~b}$ ), then the final ranking is $A_{2} \succ A_{4} \succ A_{3} \succ$ $A_{1}$, and the best one is $A_{2}$.

From the analysis above, it can be seen that the result obtained by using the similarity measure (Majumdar \& Samant, 2014) is $A_{4} \succ A_{2} \succ A_{3} \succ A_{1}$, which is consistent with that obtained by using the proposed method, while the final ranking obtained by utilising the correlation coefficient and cross entropy (Ye, 2014, 2014b) is $A_{2} \succ A_{4} \succ A_{3} \succ A_{1}$, which is different from that obtained by using the proposed method. The reason for this phenomenon is that the proposed operations and aggregation operators have been used before utilising the similarity measure (Majumdar \& Samant, 2014). However, the operations, correlation coefficient and similarity measure (Ye, 2013, 2014b) have been proved to be impractical in Section 2. Thereby, the differences were amplified in the aggregation values because of the criteria weights and the final ranking of all alternatives was influenced adversely by the similarity measure or correlation coefficient. By contrast, the proposed operations could overcome these shortcomings as were discussed in Examples 1-3. Therefore, the best alternative is $A_{4}$, which is more precise and reliable.

\section{Conclusions}

SNSs can be applied in solving problems with uncertain, imprecise, incomplete and inconsistent information that exist in scientific and engineering situations. However, as a new branch of NSs, there is not enough extant research on SNSs. In particular, the existing literatures have not proposed using the aggregation operators and the MCGDM methods for SNSs. Based on related research achievements in IFSs, the operations of SNSs were defined in this paper, and an approach to solve MCGDM problems with SNNs was proposed. Additionally, the aggregation operators of SNNWA and SNNWG, SNNOWA and SNNOWG, SNNHOWA and SNNHOWG, and GSNNWA and GSNNWG were provided. Thus, an MCGDM method was established based on the proposed operators. By using the comparison method, the ranking of all alternatives can 
be determined and the best one can easily be identified. An illustrative example demonstrated the applicability of the proposed decision-making method. Although there is no consensus on the best way to sequence SNNs, when compared to the MCDM method for SNSs (Majumdar \& Samant, 2014; Ye, 2013, 2014b), the illustrative example showed that the final result produced by the method proposed in this paper is more precise and reliable than the results produced by existing methods. Therefore, the method proposed in this paper can provide a reliable basis for SNSs. In future research, the relative measures of SNSs will be studied and applied to other fields.

\section{Acknowledgements}

The authors thank the anonymous reviewers and editors for their insightful and constructive comments and suggestions for this paper.

\section{Funding}

This work is supported by the National Natural Science Foundation of China [No. 71271218], [No. 71221061]; the Research Project of Education of Hubei [No. Q20122302]; the Science Foundation for Doctors of Hubei University of Automotive Technology [No. BK201405].

\section{Notes on contributors}

Juan-juan Peng received her M.Sc. degree in Computational Mathematics from Wuhan University of Technology, China, in 2007. She is currently a Ph.D. student in Business School, Central South University; she is also a Lecturer in School of Economics and Management, Hubei University of Automotive Technology, China. Her current research focuses on decision-making theory and application, risk management and control, and information management.

Jian-Qiang Wang received the Ph.D. degree in Management Science and Engineering from Central South University, Changsha, China, in 2005. He is currently a Professor in Business School, Central South University. His current research interests include decision-making theory and application, risk management and control, and information management.

Jing Wang received her M.Sc. degree in Information Engineering from University of Osnabrueck, Germany, in 2006. She is currently a Ph.D. student in Business School, Central South University; she is also a Lecturer in International College, Central South University of Forestry and Technology, China. Her current research focuses on decision-making theory and application, risk management and control, and information management.

Hong-Yu Zhang received her Ph.D. degree in Management Science and Engineering from Business School, Central South University, Changsha, China, in 2009. She is currently an Associated Professor in Business School, Central South University. Her research interests include the area of information management and its applications in production operations. Her current research focuses on remanufacturing production management and decisionmaking theory.
Xiao-hong Chen received her Ph.D in Tokyo University of Technology, Japan, in 1999. She is currently a professor at the School of Business, Central South University, Changsha, China. Her current research interests lie in the field of decision theory \& method, decision support system, resource-saving and environment-friendly society. He has published in several journals, including Decision Support system, Expert Systems with Applications, etc.

\section{ORCID}

Jian-qiang Wang (ㄱ) http://orcid.org/0000-0001-7668-4881 Jing Wang (1) http://orcid.org/0000-0002-2407-5985

Hong-yu Zhang (ㅇttp://orcid.org/0000-0001-5142-5277

Xiao-hong Chen (10 http://orcid.org/0000-0003-3919-5215

\section{References}

Atanassov, K. (1986). Intuitionistic fuzzy sets. Fuzzy Sets and Systems, 20(1), 87-96.

Atanassov, K. (1994). New operations defined over the intuitionistic fuzzy sets. Fuzzy Sets and Systems, 61(2), 137-142.

Atanassov, K. (1999). Intuitionistic fuzzy sets. Heidelberg: Springer.

Atanassov, K. (2000). Two theorems for intuitionistic fuzzy sets. Fuzzy Sets and Systems, 110(2), 267-269.

Atanassov, K.T., \& Gargov, G. (1989). Interval valued intuitionistic fuzzy sets. Fuzzy Sets and Systems, 31(3), 343-349.

Beliakov, G., Bustince, H., Goswami, D.P., Mukherjee, U.K., \& Pal, N.R. (2011). On averaging operators for Atanassov's intuitionistic fuzzy sets. Information Sciences, 181(6), 11161124.

Beliakov, G., Pradera, A., \& Calvo, T. (2007). Aggregation functions: A guide for practitioners. Heidelberg, Berlin, New York: Springer.

Bellman, R., \& Zadeh, L.A. (1970). Decision making in a fuzzy environment. Management Science, 17(4), 141164.

Bustince, H., \& Burillo, P. (1996). Vague sets are intuitionistic fuzzy sets. Fuzzy Sets and Systems, 79(3), 403-405.

Chaira, T. (2010). Intuitionistic fuzzy set approach for color region extraction. Journal of Scientific \& Industrial Research, 69, 426-432.

Chaira, T. (2011). A novel intuitionistic fuzzy C means clustering algorithm and its application to medical images. Applied Soft Computing, 11(2), 1711-1717.

Chen, Y.T. (2010). A outcome-oriented approach to multicriteria decision analysis with intuitionistic fuzzy optimistic/pessimistic operators. Expert Systems with Applications, 37(12), 7762-7774.

Gau, W.L., \& Buehrer, D. J. (1993). Vague sets, IEEE transactions on systems. IEEE Transactions on Systems, Man, and Cybernetics, 23(2), 610-614.

Joshi, B.P., \& Kumar, S. (2012). Fuzzy time series model based on intuitionistic fuzzy sets for empirical research in stock market. International Journal of Applied Evolutionary Computation, 3(4), 71-84.

Klement, E.P., \& Mesiar, R. (Eds.). (2005). Logical, algebraic, analytic, and probabilistic aspects of triangular norms. New York, NY: Elsevier.

Klir, G., \& Yuan, B. (1995). Fuzzy sets and fuzzy logic: Theory and applications. Upper Saddle River, NJ: Prentice Hall.

Li, L., Yang, J., \& Wu, W. (2005). Intuitionistic fuzzy hopfield neural network and its stability. Expert Systems Applications, 129, 589-597. 
Liu, H.W., \& Wang, G.J. (2007). Multi-criteria methods based on intuitionistic fuzzy sets. European Journal Operational Research, 179(1), 220-233.

Lupiáñez, F.G. (2009). Interval neutrosophic sets and topology. Kybernetes, 38(3/4), 621-624.

Majumdar, P., \& Samant, S.K. (2014). On similarity and entropy of neutrosophic sets. Journal of Intelligent and fuzzy Systems, $26,1245-1252$.

Nguyen, H.T., \& Walker, R.A. (1997). A first course in fuzzy logic. Boca Raton, FL: CRC press.

Pedrycz, W. (1990). Fuzzy sets in pattern recognition: Methodology and methods. Pattern Recognition, 23(1-2), 121-146.

Rivieccio, U. (2008). Neutrosophic logics: Prospects and problems. Fuzzy sets and systems, 159(14), 1860-1868.

Shinoj, T.K., \& Sunil, J.J. (2012). Intuitionistic fuzzy multisets and its application in medical diagnosis. International Journal of Mathematical and Computational Sciences, 6, 34-37.

Smarandache, F. (1999). A unifying field in logics. Neutrosophy: Neutrosophic probability, set and logic. Rehoboth, DE: American Research Press.

Smarandache, F. (2003). A unifying field in logics neutrosophic logic. Neutrosophy, neutrosophic set, neutrosophic probability. (3rd ed.). Xiquan, Phoenix: American Research Press.

Sotirov, S., Sotirova, E., \& Orozova, D. (2009). Neural network for defining intuitionistic fuzzy sets in e-learning. NIFS, 15, $33-36$.

Torra, V. (2010). Hesitant fuzzy sets. International Journal of Intelligent Systems, 25, 529-539.

Torra, V., \& Narukawa, Y. (2009). On hesitant fuzzy sets and decision. In The 18th IEEE International Conference on Fuzzy Systems (pp. 1378-1382). Jeju Island, Korea.

Wang, H., Smarandache, F., Zhang, Y.Q., \& Sunderraman, R. (2005). Interval neutrosophic sets and logic: Theory and applications in computing. Phoenix, AZ: Hexis.

Wang, H., Smarandache, F., Zhang, Y.Q., \& Sunderraman, R. (2010). Single valued neutrosophic sets. Multispace and Multistructure, 4, 410-413.

Wang, W.Z., \& Liu, X.W (2011). Intuitionistic fuzzy geometric aggregation operators based on Einstein operations. International Journal of Intelligent Systems, 26(11), 1049-1075.
Wang, W.Z., \& Liu, X.W. (2012). Intuitionistic fuzzy information aggregation using Einstein operations. IEEE Transactions on Fuzzy Systems, 20(5), 923-938.

$\mathrm{Xu}, \mathrm{Z}$.S. (2007). Intuitionistic fuzzy aggregation operations. IEEE Transactions on Fuzzy Systems, 15(6), 1179-1187.

$\mathrm{Xu}$, Z.S. (2008). Dependent uncertain ordered weighted aggregation operators. Information Fusion, 9(2), 310-316.

$\mathrm{Xu}, \mathrm{Z} . \mathrm{S}$. (2010). Choquet integrals of weighted intuitionistic fuzzy information. Information Sciences, 180(5), 726-736.

$\mathrm{Xu}, \mathrm{Z}$.S. (2012). Intuitionistic fuzzy multiattribute decision making: An interactive method. IEEE Transactions on Fuzzy Systems, 20(3), 514-525.

Yager, R. (2009). OWA aggregation of intuitionistic fuzzy sets. International Journal of General Systems, 38(6), 617-641.

Yager, R.R. (1977). Multiple objective decision-making using fuzzy sets. International Journal of Man-Machine Studies, 9(4), 375-382.

Ye, J. (2013). Multicriteria decision-making method using the correlation coefficient under single-value neutrosophic environment. International Journal of General Systems, 42(4), 386-394.

Ye, J. (2014a). A multicriteria decision-making method using aggregation operators for simplified neutrosophic sets. Journal of Intelligent and fuzzy Systems, 26(5), 2459-2466.

Ye, J. (2014b). Single valued neutrosophic cross-entropy for multicriteria decision making problems. Applied mathematical modeling, 38(3), 1170-1175.

Ye, J. (2014c). Similarity measures between interval neutrosophic sets and their applications in multicriteria decision-making. Journal of Intelligent and fuzzy Systems, 26(1, 165-172.

Zadeh, L.A. (1965). Fuzzy sets. Information and Control, 8(3), 338-356.

Zadeh, L.A. (1968). Probability measures of fuzzy events. Journal of Mathematical Analysis and Applications, 23(2), 421-427.

Zadeh, L.A. (1975). Fuzzy logic and approximate reasoning. Synthese, 30(3-4), 407-428.

Zeng, S.Z., \& Su, W.H. (2011). Intuitionistic fuzzy ordered weighted distance operator. Knowledge-based Systems, 24(8), $1224-1232$.

Zhi, P., \& Li, Z. (2012). A novel approach to multi-attribute decision making based on intuitionistic fuzzy sets. Expert Systems with Applications, 39(3), 2560-2566. 